Luis Alvarez - Jukka Virtanen

\title{
ON THE OPTIMAL STOCHASTIC IMPULSE CONTROL OF LINEAR DIFFUSIONS
}

Sarja Keskustelua ja raportteja/

Series Discussion and Working Papers

10:2004

ISBN: 951-564-221-3 (PDF) 951-564-220-5 (nid.)

ISSN: 1459-7632 (PDF) 0357-4687 (nid.) 


\section{ABSTRACT}

We consider a class of stochastic impulse control problems of linear diffusions arising in studies considering the determination of optimal dividend policies and in studies analyzing the optimal management of renewable resources. We derive a set of weak conditions guaranteeing both the existence and uniqueness of the optimal policy and its value by relying on a combination of the classical theory of diffusions, stochastic calculus, and ordinary nonlinear programming techniques. We also analyze two associated stochastic control problems and establish a general ordering for both the values and the marginal values of the considered stochastic control problems. 



\section{CONTENTS}

1 INTRODUCTION $\ldots \ldots \ldots \ldots \ldots \ldots \ldots \ldots \ldots$

2 THE IMPULSE CONTROL PROBLEM . . . . . . . . . . . . . 11

2.1 GENERAL SETUP . . . . . . . . . . . . . . . . 11

2.2 THE IMPULSE CONTROL PROBLEM . . . . . . . . . . . . 12

3 AUXILIARY RESULTS . . . . . . . . . . . . . . . . . 15

3.1 SOME ASSOCIATED FUNCTIONALS . . . . . . . . . . 15

3.2 THE ASSOCIATED SINGULAR CONTROL PROBLEM . . 20

3.3 THE ASSOCIATED OPTIMAL STOPPING PROBLEM . . . 25

4 OPTIMAL IMPULSE CONTROL POLICY . . . . . . . . . . . 29

4.1 NECESSARY CONDITIONS . . . . . . . . . . . . . . 29

4.2 EXISTENCE AND SUFFICIENCY . . . . . . . . . . . 31

5 ILLUSTRATION:

CONTROLLED GEOMETRIC BROWNIAN MOTION . . . . . . 39 



\section{INTRODUCTION}

A stochastic impulse control policy can typically be characterized by two factors: the random dates at which the considered policies are exercised and the size of the applied policies. This characterization naturally indicates that the timing and the size of an impulse control are factors which can be separately studied depending on the precise nature of the considered class of applications. For example, in most forest economic applications of stochastic impulse control the implemented impulse size is typically exogenously given through the exogenously determined generic initial state at which the underlying stochastic process is restarted after the forest has been harvested (see, for example, 3, 4, $5,6,26$, and 27). On the other hand, most capital theoretic and cash flow management applications of impulse control are based on models where both the timing and the size of an admissible impulse policy have to be simultaneously determined (see, for example, 7, 8, 13, 19, 24; see also 17 for an excellent survey on stochastic impulse control applications in finance). Given the applicability of stochastic impulse control it is not surprising that the mathematical analysis of such problems is well-established (see, for example, 11, 12, 15, $18,20,22$; see also 9 for a seminal textbook on quasi-variational inequalities and impulse control). In most cases the analysis of the impulse control problem is based on a combination of dynamic programming techniques and quasi-variational inequalities. Even though that approach is general and applies in the multidimensional case as well, it typically results into functional inequalities which, depending on the nature of the considered problem, may be relatively difficult to analyze and in that way difficult to interpret in terms of the particular application.

Given the arguments mentioned above, we consider in this study a class of stochastic impulse control problems where the decision maker has to choose both the timing and the size of the optimal policy affecting the dynamics of the underlying linear time-homogenous diffusion process. We generalize the analysis of the study 7 in two ways. First, instead of relying on a simple linear and state-independent exercise payoff, we introduce a state-dependent and potentially non-linear cash flow term measuring the revenue flow accrued from continuing operation (in forest economics this flow term is typically interpreted as the flow of returns accrued from amenity services; cf. 5). This extension is of interest, since as our analysis clearly demonstrates, in the presence of a 
state-dependent and potentially non-linear cash flow no strong concavity requirements are needed in order to guarantee both the existence and uniqueness of an optimal policy (which is in sharp contrast with the findings of the linear state-independent exercise payoff case studied in 7). Second, in order to model the potential imperfect controllability of the underlying stochastic dynamics, we also consider situations where an arbitrary admissible impulse may result into a jump discontinuity which is either greater or smaller than the size of the actual impulse (such configurations typically arise in models considering either the effects of taxation or the effects of financial frictions on rational cash flow management). Although this imperfection is modelled as a linear function of the applied impulse control policy, it has a profound impact on both the optimal policy and its value since it affects the required rate of return and, therefore, the marginal value of the optimal policy in a non-linear way (put somewhat differently, the linearly modelled imperfection has an nonlinear impact on the associated boundary value problems).

Instead of analyzing the considered class of stochastic impulse control problems directly via dynamic programming techniques and quasi-variational inequalities, we follow the approach introduced in 3 and 4 and first derive an associated class of iteratively defined Markovian functionals modelling the value accrued from applying a potentially suboptimal stochastic impulse control policy characterized by a sequence of constant-sized impulses exerted every time the underlying diffusion hits a predetermined and constant exercise threshold. By relying on standard nonlinear programming techniques we state the ordinary first order necessary conditions characterizing the exercise threshold and impulse size maximizing the value of the associated class of Markovian functionals. We then present a set of relatively weak sufficient conditions under which an optimal pair satisfying the necessary conditions exists and is unique and under which this solution actually characterizes both the size of the optimal impulse and the threshold at which the irreversible policy should be optimally exerted. In accordance with these observations, we then find that given the policy mentioned above the iteratively defined Markovian functional actually constitutes the value of the optimal stochastic impulse control.

We also consider two associated stochastic control problems (namely, a singular stochastic control and an optimal stopping problem) and analyze the boundary value problem connecting these values. As intuitively is clear, we find that the value of the associated singular stochastic control problem domi- 
nates the value of the stochastic impulse control problem which, in turn, dominates the value of the associated optimal stopping problem. Somewhat surprisingly, we also find that the same ordering is satisfied by the marginal values as well. More precisely, we establish the marginal value of the associated singular stochastic control problem dominates the marginal value of the stochastic impulse control problem which, in turn, dominates the marginal value of the associated optimal stopping problem. Thus, our results extend the findings of 7 by demonstrating that the positivity of the relationship between the (marginal) value and the flexibility of the admissible policy is satisfied also in the presence of a state-dependent and potentially non-linear cash flow. In economic terms, our findings unambiguously prove that increased policy flexibility does not only increase the value of the optimal admissible policy, it also increases the rate at which this value grows.

The contents of this study are as follows. In section two we present the considered class of stochastic impulse control problems. In section three we then state a set of auxiliary results and analyze the two associated stochastic control problems. In section four we then analyze the considered stochastic impulse control problem and state our main results. Finally, our results are explicitly illustrated in section five in a model based on geometric Brownian motion. 



\section{THE IMPULSE CONTROL PROBLEM}

\subsection{GENERAL SETUP}

It is our purpose in this study to analyze a class of stochastic impulse control problems of linear diffusions arising in many financial and economical applications of stochastic control theory. In order to accomplish this task, let $\left(\Omega, \mathcal{F},\left\{\mathcal{F}_{t}\right\}_{t \geq 0}, \mathbf{P}\right)$ denote a complete filtered probability space satisfying the usual conditions and assume that the dynamics of the underlying controlled diffusion process are given by the generalized Itô equation

$$
X_{t}^{\nu}=x+\int_{0}^{t} \mu\left(X_{s}^{\nu}\right) d s+\int_{0}^{t} \sigma\left(X_{s}^{\nu}\right) d W_{s}-\sum_{\tau_{k} \leq t} \beta \zeta_{k}, 0 \leq t \leq \tau_{0}^{\nu}
$$

where $\beta>0$ is exogenously given constant, $\tau_{0}^{\nu}=\inf \left\{t \geq 0 \mid X_{t}^{\nu} \leq 0\right\}$ denotes the possibly finite first exit date from the state-space $\mathbb{R}_{+}$of the controlled diffusion process and $\mu: \mathbb{R}_{+} \rightarrow \mathbb{R}$ and $\sigma: \mathbb{R}_{+} \rightarrow \mathbb{R}_{+}$are known sufficiently smooth mappings (at least continuous) guaranteeing the existence of a solution for the stochastic differential equation

$$
d X_{t}=\mu\left(X_{t}\right) d t+\sigma\left(X_{t}\right) d W_{t}, \quad X_{0}=x,
$$

characterizing the dynamics of the underlying diffusion in the absence of interventions (cf. 10, pp. 46-47). It is at this point worth emphasizing that the parameter $\beta$ can be interpreted as a measure of the imperfect controllability of the underlying stochastic dynamics, since an arbitrary admissible impulse results into a jump discontinuity which is either greater or smaller than the size of the actual impulse whenever $\beta \neq 1$. As in 22, an impulse control for the system (2.1) is a potentially infinite joint sequence $\nu=\left\{\left(\tau_{k}, \zeta_{k}\right)\right\}_{k=1}^{N}, N \leq \infty$, where $\left\{\tau_{k}\right\}_{k=1}^{N}$ denotes an increasing sequence of $\mathcal{F}_{t}$-stopping times for which $\tau_{1}>0$ and $\left\{\zeta_{k}\right\}_{k=1}^{N}$ denotes a sequence of non-negative impulses exerted at the corresponding intervention dates $\left\{\tau_{k}\right\}_{k=1}^{N}$, respectively. We denote as $\mathcal{V}$ the class of admissible impulse controls $\nu$ and assume that $\tau_{k} \rightarrow \tau_{0}^{\nu}$ almost surely for all $\nu \in \mathcal{V}$ and $x \in \mathbb{R}_{+}$. Furthermore, in line with most financial and economical applications, we assume that the upper boundary $\infty$ is natural and the lower boundary 0 is either natural, exit or regular for the controlled diffusion in the absence of interventions. Whenever 0 is regular for $X_{t}$, it is assumed to be killing (in line with the concept of liquidation). As usually, we 
denote as

$$
\mathcal{A}=\frac{1}{2} \sigma^{2}(x) \frac{d^{2}}{d x^{2}}+\mu(x) \frac{d}{d x}
$$

the differential operator associated with the underlying diffusion $X_{t}$.

\subsection{THE IMPULSE CONTROL PROBLEM}

Given the stochastic dynamics in (2.1) and the assumptions presented above on the dynamics of the controlled system, define the expected cumulative net present value of the revenues from the present up to a potentially infinite future as

$$
J_{c}^{\nu}(x)=\mathbf{E}_{x}\left[\int_{0}^{\tau_{0}^{\nu}} e^{-r s} \pi\left(X_{s}^{\nu}\right) d s+\sum_{k=1}^{N} e^{-r \tau_{k}}\left(\lambda \zeta_{k}-c\right)\right],
$$

where $\lambda>0$ is an exogenously given constant, $c>0$ is a known constant measuring a lump-sum sunk cost associated with the irreversible policy, and $\pi: \mathbb{R}_{+} \rightarrow \mathbb{R}_{+}$is a given continuous, non-decreasing and non-negative mapping measuring the revenue flow accrued from continuing the operation. This type of objective functional arise frequently in studies considering rational cash flow management (optimal dividend policy) and in studies considering the rational harvesting of renewable resources.

Given the definition of $J_{c}^{\nu}(x)$ we plan to study the stochastic impulse control problem

$$
V_{c}(x)=\sup _{\nu \in \mathcal{V}} J_{c}^{\nu}(x), x \in \mathbb{R}_{+}
$$

and to determine an admissible impulse control $\nu^{*}$ for which the maximum $J_{c}^{\nu^{*}}(x)=V_{c}(x)$ is attained for all $x \in \mathbb{R}_{+}$. In order to proceed in the analysis of the considered class of stochastic control problems, we first establish the following verification theorem.

Lemma 2.1. Assume that there is a mapping $F: \mathbb{R}_{+} \mapsto \mathbb{R}_{+}$satisfying the conditions

(a) $\left(R_{r} \pi\right)(x)-F(x)$ is r-subharmonic for the diffusion $X_{t}$, and

(b) $F(x)$ satisfies for all $x \in \mathbb{R}_{+}$the inequality

$$
F(x) \geq \sup _{\beta \zeta \in[0, x]}[\lambda \zeta-c+F(x-\beta \zeta)] .
$$


Then, $F(x) \geq V_{c}(x)$ for all $x \in \mathbb{R}_{+}$.

Proof. Let $\nu \in \mathcal{V}$ be an admissible stochastic impulse control. Since $\left\{\tau_{j}\right\}_{j \in \mathbb{N}}$ is an increasing sequence of stopping times, we first observe that the assumed $r$-subharmonicity of the mapping $\left(R_{r} \pi\right)(x)-F(x)$ implies that

$$
\begin{aligned}
& \mathbf{E}\left[e^{-r \tau_{j+1}}\left(\left(R_{r} \pi\right)\left(X_{\tau_{j+1}-}^{\nu}\right)-F\left(X_{\tau_{j+1}-}^{\nu}\right)\right) \mid \mathcal{F}_{\tau_{j}}\right] \geq \\
& e^{-r \tau_{j}}\left(\left(R_{r} \pi\right)\left(X_{\tau_{j}}^{\nu}\right)-F\left(X_{\tau_{j}}^{\nu}\right)\right) .
\end{aligned}
$$

Applying Dynkin's theorem to the mapping $\left(R_{r} \pi\right)(x)$ now yields

$$
\begin{aligned}
\mathbf{E}\left[e^{-r \tau_{j+1}}\left(R_{r} \pi\right)\left(X_{\tau_{j+1}-}^{\nu}\right) \mid \mathcal{F}_{\tau_{j}}\right] & =e^{-r \tau_{j}}\left(R_{r} \pi\right)\left(X_{\tau_{j}}^{\nu}\right) \\
& -\mathbf{E}_{\mathcal{F}_{\tau_{j}}} \int_{\tau_{j}}^{\tau_{j+1-}} e^{-r s} \pi\left(X_{s}^{\nu}\right) d s
\end{aligned}
$$

implying that inequality (2.6) can be re-expressed as

$$
e^{-r \tau_{j}} F\left(X_{\tau_{j}}^{\nu}\right)-\mathbf{E}\left[e^{-r \tau_{j+1}} F\left(X_{\tau_{j+1}-}^{\nu}\right) \mid \mathcal{F}_{\tau_{j}}\right] \geq \mathbf{E}_{\mathcal{F}_{\tau_{j}}} \int_{\tau_{j}}^{\tau_{j+1-}} e^{-r s} \pi\left(X_{s}^{\nu}\right) d s .
$$

Taking expectations and invoking the tower property of conditional expectations then yields

$$
\mathbf{E}_{x}\left[e^{-r \tau_{j}} F\left(X_{\tau_{j}}^{\nu}\right)\right]-\mathbf{E}_{x}\left[e^{-r \tau_{j+1}} F\left(X_{\tau_{j+1}-}^{\nu}\right)\right] \geq \mathbf{E}_{x} \int_{\tau_{j}}^{\tau_{j+1-}} e^{-r s} \pi\left(X_{s}^{\nu}\right) d s .
$$

Letting $\tau_{0}=0$, summing terms from $j=0$ to $j=n \wedge N$, and applying the nonnegativity of the mapping $F(x)$ results in

$$
F(x) \geq \sum_{j=1}^{n \wedge N} e^{-r \tau_{j}} \mathbf{E}_{x}\left[F\left(X_{\tau_{j-}}^{\nu}\right)-F\left(X_{\tau_{j}}^{\nu}\right)\right]+\mathbf{E}_{x} \int_{0}^{\tau_{n \wedge N+1-}} e^{-r s} \pi\left(X_{s}^{\nu}\right) d s .
$$

Since $X_{\tau_{j}}=X_{\tau_{j}-}-\beta \zeta_{j}$ for any admissible strategy and $F(x)$ satisfies the quasi-variational inequality $F(x) \geq \sup _{\beta \zeta \in[0, x]}[\lambda \zeta-c+F(x-\beta \zeta)]$ for all $\in \mathbb{R}_{+}$we find that

$$
F(x) \geq \mathbf{E}_{x}\left[\int_{0}^{\tau_{n \wedge N+1-}} e^{-r s} \pi\left(X_{s}^{\nu}\right) d s+\sum_{j=1}^{n \wedge N} e^{-r \tau_{j}}\left(\lambda \zeta_{j}-c\right)\right] .
$$


Letting $n \rightarrow \infty$ and invoking dominated convergence then finally implies that

$$
F(x) \geq \mathbf{E}_{x}\left[\int_{0}^{\tau_{0}^{\nu}} e^{-r s} \pi\left(X_{s}^{\nu}\right) d s+\sum_{j=1}^{N} e^{-r \tau_{j}}\left(\lambda \zeta_{j}-c\right)\right] .
$$

Since this inequality is valid for any admissible impulse control, it has to be valid for the optimal as well from which the alleged result follows.

Lemma 2.1 states a set of considerably weak sufficient conditions which can be applied in the verification of the optimality of a value attained by applying an admissible policy. An interesting implication of Lemma 2.1 stating a set of more easily applicable sufficient conditions is now summarized in the following.

Corollary 2.2. Assume that the mapping $F: \mathbb{R}_{+} \mapsto \mathbb{R}_{+}$satisfies the conditions $F \in C^{1}\left(\mathbb{R}_{+}\right) \cap C^{2}\left(\mathbb{R}_{+} \backslash \mathcal{D}\right)$, where $\mathcal{D}$ is a set of measure zero and $F^{\prime \prime}(x \pm)<\infty$ for all $x \in \mathcal{D}$. Assume also that $F(x)$ satisfies the quasi-variational inequality (2.5) for all $x \in \mathbb{R}_{+}$and the variational inequality $(\mathcal{A} F)(x)-r F(x)+\pi(x) \leq$ 0 for all $x \notin \mathcal{D}$. Then, $F(x) \geq V_{c}(x)$ for all $x \in \mathbb{R}_{+}$.

Proof. As was established in Theorem D.1. in 23 (pp. 315-318) the conditions of our corollary guarantee that there a sequence $\left\{F_{n}\right\}_{n=1}^{\infty}$ of mappings $F_{n} \in$ $C^{2}\left(\mathbb{R}_{+}\right)$such that

(i) $F_{n} \rightarrow F$ uniformly on compact subsets of $\mathbb{R}_{+}$, as $n \rightarrow \infty$;

(ii) $\left(\mathcal{A} F_{n}\right)-r F_{n} \rightarrow(\mathcal{A F})-r F$ uniformly on compact subsets of $\mathbb{R}_{+} \backslash \mathcal{D}$, as $n \rightarrow \infty$;

(iii) $\left\{\left(\mathcal{A} F_{n}\right)-r F_{n}\right\}_{n=1}^{\infty}$ is locally bounded on $\mathbb{R}_{+}$.

Applying Itô's theorem to the mapping $(t, x) \mapsto e^{-r t} \Delta_{n}(x)$, where $\Delta_{n}(x)=$ $\left(\left(R_{r} \pi\right)(x)-F_{n}(x)\right)$, taking expectations, and reordering terms yields

$$
\begin{aligned}
e^{-r \tau_{j}} \Delta_{n}\left(X_{\tau_{j}}^{\nu}\right)=\mathbf{E}\left[e^{-r \tau_{j+1}} \Delta_{n}\left(X_{\tau_{j+1}-}^{\nu}\right)-\int_{\tau_{j}}^{\tau_{j+1-}} e^{-r s}\left(\left(\mathcal{A} F_{n}\right)\left(X_{s}^{\nu}\right)\right.\right. \\
\left.\left.-r F_{n}\left(X_{s}^{\nu}\right)+\pi\left(X_{s}^{\nu}\right)\right) d s \mid \mathcal{F}_{\tau_{j}}\right]
\end{aligned}
$$

Letting $n \rightarrow \infty$, applying Fatou's theorem, and invoking the variational inequality $(\mathcal{A} F)(x)-r F(x)+\pi(x) \leq 0$ then yields

$$
e^{-r \tau_{j}}\left(\left(R_{r} \pi\right)\left(X_{\tau_{j}}^{\nu}\right)-F\left(X_{\tau_{j}}^{\nu}\right)\right) \geq \mathbf{E}\left[e^{-r \tau_{j+1}}\left(\left(R_{r} \pi\right)\left(X_{\tau_{j+1-}}^{\nu}\right)-F\left(X_{\tau_{j+1-}}^{\nu}\right)\right) \mid \mathcal{F}_{\tau_{j}}\right] .
$$

The alleged result now follows from Lemma 2.1. 


\section{AUXILIARY RESULTS}

\subsection{SOME ASSOCIATED FUNCTIONALS}

Denote as $\mathcal{L}_{1}\left(\mathbb{R}_{+}\right)$the class of measurable mappings $f: \mathbb{R}_{+} \rightarrow \mathbb{R}$ satisfying the uniform integrability condition

$$
\mathbf{E}_{x}\left[\int_{0}^{\tau_{0}} e^{-r s}\left|f\left(X_{s}\right)\right| d s\right],
$$

where $\tau_{0}=\inf \left\{t \geq 0 \mid X_{t} \leq 0\right\}$ denotes the first, potentially infinite, exit date for the uncontrolled diffusion $X_{t}$ from the state-space $\mathbb{R}_{+}$. Given the class $\mathcal{L}_{1}\left(\mathbb{R}_{+}\right)$, define for arbitrary $f \in \mathcal{L}_{1}\left(\mathbb{R}_{+}\right)$the resolvent functional $\left(R_{r} f\right)$ : $\mathbb{R}_{+} \rightarrow \mathbb{R}$ measuring the expected cumulative present value of the cash flow $f\left(X_{t}\right)$ from the present up to the first liquidation date $\tau_{0}$ as

$$
\left(R_{r} f\right)(x)=\mathbf{E}_{x}\left[\int_{0}^{\tau_{0}} e^{-r s} f\left(X_{s}\right) d s\right] .
$$

It is a well-known fact from the literature on linear diffusions that the expected cumulative present value of a cash flow $f \in \mathcal{L}_{1}\left(\mathbb{R}_{+}\right)$can be rewritten as

$$
\left(R_{r} f\right)(x)=B^{-1} \varphi(x) \int_{0}^{x} \psi(y) f(y) m^{\prime}(y) d y+B^{-1} \psi(x) \int_{x}^{\infty} \varphi(y) f(y) m^{\prime}(y) d y,
$$

where $\psi$ is the increasing and $\varphi$ is the decreasing fundamental solution of the ordinary second-order linear differential equation $(\mathcal{A} u)=r u$ defined on the domain of the characteristic operator of the diffusion $X_{t}$ (see 10 pp. 18-20 for a throughout characterization of the associated fundamental solutions and the Green function of a linear diffusion),

$$
B=\frac{\psi^{\prime}(x)}{S^{\prime}(x)} \varphi(x)-\frac{\varphi^{\prime}(x)}{S^{\prime}(x)} \psi^{\prime}(x)
$$

denotes the constant (with respect to the scale) Wronskian determinant,

$$
S^{\prime}(x)=\exp \left(-\int^{x} \frac{2 \mu(y)}{\sigma^{2}(y)} d y\right)
$$

denotes the density of the scale function $S$ of the diffusion $X_{t}$ and

$$
m^{\prime}(x)=\frac{2}{\sigma^{2}(x) S^{\prime}(x)}
$$


denotes the density of the speed measure $m$ of the diffusion $X_{t}$.

Define now the mapping $\theta: \mathbb{R}_{+} \rightarrow \mathbb{R}$ measuring the rate at which the total revenues are appreciating as

$$
\theta(x)=\beta \pi(x)+\lambda \rho(x),
$$

where $\rho(x)=\mu(x)-r x$ measures the net appreciation rate of the underlying controlled diffusion $X_{t}$. Throughout this study we will assume that $\pi, \rho \in \mathcal{L}_{1}\left(\mathbb{R}_{+}\right)$, therefore also $\theta \in \mathcal{L}_{1}\left(\mathbb{R}_{+}\right)$. Consider now the expected cumulative present value $\left(R_{r} \theta\right)(x)$. By invoking the Greenian representation (3.1), differentiating the equation sidewise and dividing sidewise with the term $\psi^{\prime}(x)$ we find out that

$$
\frac{\left(R_{r} \theta\right)^{\prime}(x)}{\psi^{\prime}(x)}=B^{-1} \frac{\varphi^{\prime}(x)}{\psi^{\prime}(x)} \int_{0}^{x} \psi(y) \theta(y) m^{\prime}(y) d y+B^{-1} \int_{x}^{\infty} \varphi(y) \theta(y) m^{\prime}(y) d y .
$$

Now ordinary differentiation yields

$$
\frac{d}{d x}\left[\frac{\left(R_{r} \theta\right)^{\prime}(x)}{\psi^{\prime}(x)}\right]=\frac{2 S^{\prime}(x)}{\sigma^{2}(x) \psi^{\prime 2}(x)} L(x),
$$

where the functional $L: \mathbb{R}_{+} \rightarrow \mathbb{R}$ is defined as

$$
L(x)=r \int_{0}^{x} \psi(y) \theta(y) m^{\prime}(y) d y-\theta(x) \frac{\psi^{\prime}(x)}{S^{\prime}(x)} .
$$

The functional $L$ will prove to be the key ingredient when determining optimal policies in all the considered stochastic control problems. The next lemma will shed light on some of the useful properties of the functional $L$ under reasonable assumptions on the mapping $\theta$.

Lemma 3.1. Assume, that there exists a state $x^{*} \in \mathbb{R}_{+}$for which the function $\theta$ is increasing on the interval $\left(0, x^{*}\right)$ and decreasing on the interval $\left(x^{*}, \infty\right)$. Moreover, assume that $0 \leq \lim _{x \downarrow 0} \theta(x)<\infty$ and that $\lim _{x \rightarrow \infty} \theta(x)<0$. Then there exists a unique state $\hat{x}=\operatorname{argmin}\left\{\left(R_{r} \theta\right)^{\prime}(x) / \psi^{\prime}(x)\right\} \in\left(x^{*}, \infty\right)$ satisfying the condition $L(\hat{x})=0$.

Proof. For $z>x>x^{*}$ we have that

$$
\begin{aligned}
\frac{1}{r}[L(z)-L(x)] & =\int_{x}^{z} \psi(y) \theta(y) m^{\prime}(y) d y-\frac{\theta(z)}{r} \frac{\psi^{\prime}(z)}{S^{\prime}(z)}+\frac{\theta(x)}{r} \frac{\psi^{\prime}(x)}{S^{\prime}(x)} \\
& >\frac{\theta(z)}{r}\left[\frac{\psi^{\prime}(z)}{S^{\prime}(z)}-\frac{\psi^{\prime}(x)}{S^{\prime}(x)}\right]-\frac{\theta(z)}{r} \frac{\psi^{\prime}(z)}{S^{\prime}(z)}+\frac{\theta(x)}{r} \frac{\psi^{\prime}(x)}{S^{\prime}(x)} \\
& =\frac{[\theta(x)-\theta(z)]}{r} \frac{\psi^{\prime}(x)}{S^{\prime}(x)}>0,
\end{aligned}
$$


proving that $L$ is monotonously increasing on $\left(x^{*}, \infty\right)$. In the same manner, we see that whenever $z<x<x^{*}$

$$
\begin{aligned}
\frac{1}{r}[L(x)-L(z)] & =\int_{z}^{x} \psi(y) \theta(y) m^{\prime}(y) d y-\frac{\theta(x)}{r} \frac{\psi^{\prime}(x)}{S^{\prime}(x)}+\frac{\theta(z)}{r} \frac{\psi^{\prime}(z)}{S^{\prime}(z)} \\
& <\frac{[\theta(z)-\theta(x)]}{r} \frac{\psi^{\prime}(z)}{S^{\prime}(z)}<0
\end{aligned}
$$

showing that $L$ is monotonously decreasing on $\left(0, x^{*}\right)$.

Since the boundary 0 is assumed to be natural, exit or killing, we see immediately that $\lim _{x \downarrow 0} L(x) \leq 0$. Moreover, the assumed monotonicity properties of the mapping $\theta(x)$ implies that

$$
\begin{aligned}
L\left(x^{*}\right) & =r \int_{0}^{x^{*}} \psi(y) \theta(y) m^{\prime}(y) d y-\theta\left(x^{*}\right) \frac{\psi^{\prime}\left(x^{*}\right)}{S^{\prime}\left(x^{*}\right)} \\
& \leq \theta\left(x^{*}\right)\left[\frac{\psi^{\prime}\left(x^{*}\right)}{S^{\prime}\left(x^{*}\right)}-\frac{\psi^{\prime}(0)}{S^{\prime}(0)}\right]-\theta\left(x^{*}\right) \frac{\psi^{\prime}\left(x^{*}\right)}{S^{\prime}\left(x^{*}\right)}<0 .
\end{aligned}
$$

On the basis of the assumptions on the mapping $\theta$ it is evident that there exists a state $x_{0} \in\left(x^{*}, \infty\right)$ such that $\theta\left(x_{0}\right)=0$. Moreover, $\theta(x)>0$ whenever $x \in\left(0, x_{0}\right)$. Hence, the proved monotonicity of $L(x)$ and the inequality

$$
L\left(x_{0}\right)=r \int_{0}^{x_{0}} \psi(y) \theta(y) m^{\prime}(y) d y>0
$$

prove that there is a unique state $\hat{x} \in\left(x^{*}, \infty\right)$ such that $L(\hat{x})=0$.

Along with the functional $L$, two additional functionals will be of great importance in the subsequent study of the considered stochastic control problems. These functionals, which are now denoted as $I: \mathbb{R}_{+} \rightarrow \mathbb{R}$ and $J: \mathbb{R}_{+} \rightarrow \mathbb{R}$, are defined as

$$
I(x)=\frac{\beta\left(R_{r} \pi\right)^{\prime}(x)-\lambda}{\psi^{\prime}(x)}
$$

and as

$$
J(x)=\beta\left(R_{r} \pi\right)(x)-\lambda x-I(x) \psi(x) .
$$

However, as we are about to establish, the key properties of these functionals $I$ and $J$ are dictated by the behavior of $L$, the behavior of which is again, by definition, dictated by $\theta$. First note that since $\rho \in \mathcal{L}_{1}\left(\mathbb{R}_{+}\right)$the expression

$$
\frac{d}{d x}\left[\frac{\left(R_{r} \rho\right)^{\prime}(x)}{\psi^{\prime}(x)}\right]=\frac{2 S^{\prime}(x)}{\sigma^{2}(x) \psi^{\prime 2}(x)}\left[r \int_{0}^{x} \psi(y) \rho(y) m^{\prime}(y) d y-\rho(x) \frac{\psi^{\prime}(x)}{S^{\prime}(x)}\right]
$$


holds. On the other hand, since (cf. 3, Lemma 2.1)

$$
\psi^{\prime \prime}(x)=\frac{2 S^{\prime}(x)}{\sigma^{2}(x)}\left[r \int_{0}^{x} \psi(y) \rho(y) m^{\prime}(y) d y-\rho(x) \frac{\psi^{\prime}(x)}{S^{\prime}(x)}\right],
$$

we have that

$$
\frac{d}{d x}\left[\frac{\left(R_{r} \rho\right)^{\prime}(x)}{\psi^{\prime}(x)}\right]=\frac{\psi^{\prime \prime}(x)}{\psi^{\prime 2}(x)} .
$$

Consequently, we discover that

$$
\begin{aligned}
I^{\prime}(x) & =\frac{d}{d x}\left[\frac{\beta\left(R_{r} \pi\right)^{\prime}(x)-\lambda}{\psi^{\prime}(x)}\right]=\frac{d}{d x}\left[\frac{\left(R_{r} \theta\right)^{\prime}(x)}{\psi^{\prime}(x)}\right] \\
& =\frac{2 S^{\prime}(x)}{\sigma^{2}(x) \psi^{\prime 2}(x)} L(x) \lesseqgtr 0, \text { when } x \lesseqgtr \hat{x} .
\end{aligned}
$$

In other words, the fact whether the functional $I$ is increasing or decreasing is dictated by the sign of the value of the functional $L$. Secondly, ordinary differentiation yields

$$
J^{\prime}(x)=-\psi(x) I^{\prime}(x) \lesseqgtr 0, \text { when } x \gtreqless \hat{x} ;
$$

hence $L$ dictates also the monotonicity properties of $J$. Finally, invoking the Greenian representation of the resolvent $\left(R_{r} \pi\right)(x)$, we discover that

$$
\begin{aligned}
J(x) & =\beta \frac{S^{\prime}(x)}{\psi^{\prime}(x)} \int_{0}^{x} \psi(y) \pi(y) m^{\prime}(y) d y+\lambda\left(\frac{\psi(x)}{\psi^{\prime}(x)}-x\right) \\
& =\frac{S^{\prime}(x)}{\psi^{\prime}(x)} \int_{0}^{x} \psi(y) \theta(y) m^{\prime}(y) d y,
\end{aligned}
$$

since

$$
\frac{\psi(x)}{\psi^{\prime}(x)}-x=\frac{S^{\prime}(x)}{\psi^{\prime}(x)} \int_{0}^{x} \psi(y) \rho(y) m^{\prime}(y) d y
$$

(cf. 7, Lemma 3.3). To close the subsection, we present a lemma determining the boundary properties of the functionals $I$ and $J$ under assumptions of Lemma 3.1 on the mapping $\theta$.

Lemma 3.2. Assume that the conditions of lemma 3.1 are satisfied. Then $\lim _{x \downarrow 0} J(x) \geq 0, \lim _{x \downarrow 0} I(x) \geq 0, \lim _{x \rightarrow \infty} J(x)=-\infty$ and $\lim _{x \rightarrow \infty} I(x) \leq$ 0 . 
Proof. We have shown that

$$
J(x)=\frac{S^{\prime}(x)}{\psi^{\prime}(x)} \int_{0}^{x} \psi(y) \theta(y) m^{\prime}(y) d y .
$$

If 0 is attainable, then $J(0)=0$, since $\lim _{x \downarrow 0} \frac{\psi^{\prime}(x)}{S^{\prime}(x)}>0$. On the other hand, if 0 is unattainable, then $\lim _{x \downarrow 0} \frac{\psi^{\prime}(x)}{S^{\prime}(x)}=0$. In this case, invoking L'Hospitals rule yields

$$
\lim _{x \downarrow 0} J(x)=\lim _{x \downarrow 0} \frac{\psi(x) \theta(x) m^{\prime}(x)}{\frac{d}{d x}\left[\frac{\psi^{\prime}(x)}{S^{\prime}(x)}\right]}=\lim _{x \downarrow 0} \frac{\psi(x) \theta(x) m^{\prime}(x)}{r \psi(x) m^{\prime}(x)}=\lim _{x \downarrow 0} \frac{\theta(x)}{r} \geq 0 .
$$

To prove the alleged behavior of $J$ at infinity, note that

$$
\lim _{x \rightarrow \infty} \int_{0}^{x} \psi(y) \theta(y) m^{\prime}(y) d y=-\infty
$$

and that $\lim _{x \rightarrow \infty} \frac{\psi^{\prime}(x)}{S^{\prime}(x)}=\infty$. Thus

$$
\lim _{x \rightarrow \infty} J(x)=\lim _{x \rightarrow \infty} \frac{\theta(x)}{r}=-\infty .
$$

We showed in the Lemma 3.1 that the state $\hat{x}$ lies in the interval $\left(x^{*}, \infty\right)$, i.e. where $\theta$ is monotonously decreasing. Hence for $x>\hat{x}$, we have that

$$
\begin{aligned}
I(x) & =B^{-1} \frac{\varphi^{\prime}(x)}{\psi^{\prime}(x)}\left[\frac{\theta(\hat{x})}{r} \frac{\psi^{\prime}(\hat{x})}{S^{\prime}(\hat{x})}+\int_{\hat{x}}^{x} \psi(y) \theta(y) m^{\prime}(y) d y\right]+B^{-1} \int_{x}^{\infty} D(y) d y \\
& \leq B^{-1} \frac{\varphi^{\prime}(x)}{\psi^{\prime}(x)}\left[\frac{\theta(\hat{x})}{r} \frac{\psi^{\prime}(\hat{x})}{S^{\prime}(\hat{x})}+\frac{\theta(\hat{x})}{r}\left(\frac{\psi^{\prime}(x)}{S^{\prime}(x)}-\frac{\psi^{\prime}(\hat{x})}{S^{\prime}(\hat{x})}\right)\right]+B^{-1} \int_{x}^{\infty} D(y) d y \\
& =B^{-1} \frac{\varphi^{\prime}(x)}{S^{\prime}(x)} \frac{\theta(\hat{x})}{r}+B^{-1} \int_{x}^{\infty} D(y) d y,
\end{aligned}
$$

where $D(y):=\varphi(y) \theta(y) m^{\prime}(y)$. By letting $x$ tend to infinity in the inequality above, we discover that

$$
\lim _{x \rightarrow \infty} I(x) \leq 0,
$$

since $\lim _{x \rightarrow \infty} \frac{\varphi^{\prime}(x)}{S^{\prime}(x)}=0$. The property $\lim _{x \downarrow 0} I(x) \geq 0$ is still left to prove. In order to prove this, observe that the condition $I(x) \geq I(\hat{x})$ implies that

$$
\frac{\left(R_{r} \theta\right)^{\prime}(x)}{S^{\prime}(x)} \geq I(\hat{x}) \frac{\psi^{\prime}(x)}{S^{\prime}(x)}
$$


By continuity, this means that

$$
\lim _{x \downarrow 0} \frac{\left(R_{r} \theta\right)^{\prime}(x)}{S^{\prime}(x)} \geq \lim _{x \downarrow 0} I(\hat{x}) \frac{\psi^{\prime}(x)}{S^{\prime}(x)}=0 ;
$$

hence $\lim _{x \downarrow 0}\left(R_{r} \theta\right)^{\prime}(x) \geq 0$. Now the desired result

$$
\lim _{x \downarrow 0} I(x)=\lim _{x \downarrow 0} \frac{\left(R_{r} \theta\right)^{\prime}(x)}{\psi^{\prime}(x)} \geq 0
$$

follows, since $\psi^{\prime}(x)>0$.

\subsection{THE ASSOCIATED SINGULAR CONTROL PROBLEM}

Before proceeding to the analysis of the stochastic impulse control problem, we first consider an associated singular stochastic control problem and illustrate how the two stochastic control problems are connected. In order to accomplish this task, consider the associated controlled diffusion process $X_{t}^{Z}$ described on $\mathbb{R}_{+}$by the generalized Itô stochastic differential equation

$$
d X_{t}^{Z}=\mu\left(X_{t}^{Z}\right) d t+\sigma\left(X_{t}^{Z}\right) d W_{t}-\beta d Z_{t}, X_{0}^{Z}=x,
$$

where the process $Z_{t}$ is an admissible (bounded variation) control, meaning a non-negative, non-decreasing, right-continuous and $\left\{\mathcal{F}_{t}\right\}$-adapted process. We denote the class of such processes as $\Lambda$ and assume that $\mu$ and $\sigma$ satisfy the same regularity conditions as in the impulse control case. Given these assumptions, we will next consider the associated singular control problem

$$
K(x)=\sup _{Z \in \Lambda} \mathbf{E}_{x}\left[\int_{0}^{\tau_{0}^{Z}} e^{-r s}\left(\pi\left(X_{s}^{Z}\right) d s+\lambda d Z_{s}\right)\right],
$$

where $\tau_{0}^{Z}=\inf \left\{t \geq 0 \mid X_{t}^{Z} \leq 0\right\}$ denotes the first exit date of the controlled diffusion from $\mathbb{R}_{+}$. It is worth observing that applying the generalized Itô theorem to the linear mapping $x \mapsto \lambda x / \beta$ yields

$$
\mathbf{E}_{x} \int_{0}^{\tau_{N}} e^{-r s} \lambda d Z_{s}=\frac{\lambda x}{\beta}+\mathbf{E}_{x} \int_{0}^{\tau_{N}} e^{-r s} \frac{\lambda}{\beta} \rho\left(X_{s}^{Z}\right) d s-\mathbf{E}_{x}\left[e^{-r \tau_{N}} \frac{\lambda}{\beta} X_{\tau_{N}}^{Z}\right]
$$

where $\tau_{N}=\tau_{0}^{Z} \wedge N \wedge \inf \left\{t \geq 0 \mid X_{t}^{Z} \geq N\right\}$ is an almost surely finite stopping time. The non-negativity of the controlled process then results by letting $N$ 
tend to infinity and invoking monotone convergence to the inequality

$$
K(x) \leq \beta^{-1}\left[\lambda x+\sup _{Z \in \Lambda} \mathbf{E}_{x} \int_{0}^{\tau_{0}^{Z}} e^{-r s} \theta\left(X_{s}^{Z}\right) d s\right] .
$$

It is clear that if the implemented admissible policy satisfies the condition $\lim _{N \rightarrow \infty} \mathbf{E}_{x}\left[e^{-r \tau_{N}} X_{\tau_{N}}^{Z}\right]=0$ then the inequality (3.5) becomes an equality. In that case the value of the optimal policy can be decomposed into a part measuring the value of the instantaneous liquidation policy and the expected cumulative present value of the future revenues accrued from postponing the immediate liquidation of the underlying process.

In the next lemma we will establish the value and the optimal policy for the problem (3.4). These results will later turn out to be useful in the analysis of the impulse control problem (2.4) as well.

Lemma 3.3. Assume, that the conditions of the Lemma 3.1 are met. Then optimal singular stochastic control reads as

$$
Z_{t}= \begin{cases}(x-\hat{x})^{+} & t=0 \\ \mathcal{L}(t, \hat{x}) & t>0\end{cases}
$$

where the threshold $\hat{x} \in\left(x^{*}, \infty\right)$ is the unique root of the first-order condition $L(\hat{x})=0$. Moreover, the value of the optimal policy reads as

$$
K(x)= \begin{cases}\beta^{-1}\left(\lambda x+\frac{\theta(\hat{x})}{r}\right) & x \geq \hat{x} \\ \left(R_{r} \pi\right)(x)-\beta^{-1} I(\hat{x}) \psi(x) & x<\hat{x}\end{cases}
$$

implying that the marginal value of the optimal policy can be expressed as

$$
\begin{aligned}
K^{\prime}(x) & =\left(R_{r} \pi\right)^{\prime}(x)+\beta^{-1} \psi^{\prime}(x) \sup _{y \geq x}\left[\frac{\lambda-\beta\left(R_{r} \pi\right)^{\prime}(y)}{\psi^{\prime}(y)}\right] \\
& = \begin{cases}\lambda \beta^{-1} & x \geq \hat{x} \\
\left(R_{r} \pi\right)^{\prime}(x)-\beta^{-1} I(\hat{x}) \psi^{\prime}(x) & x<\hat{x} .\end{cases}
\end{aligned}
$$

Proof. Denote the proposed value function as $K_{p}(x)$. It is clear that $K_{p}(x) \leq$ $K(x)$, since $K_{p}(x)$ is attained by applying the admissible local time push (i.e. reflection at $\hat{x}$ ) policy (3.6). In order to establish the opposite inequality, we 
first observe that the proposed value function is twice continuously differentiable on $\mathbb{R}_{+}$. Moreover, ordinary differentiation yields that

$$
K_{p}^{\prime}(x)= \begin{cases}\lambda \beta^{-1} & x \geq \hat{x} \\ \beta^{-1}\left[\lambda+\psi^{\prime}(x)(I(x)-I(\hat{x}))\right] & x<\hat{x} .\end{cases}
$$

Since the state $\hat{x}$ is the global minimum of the functional $I$, we find that $K_{p}^{\prime}(x) \geq \lambda \beta^{-1}$ for all $x \in \mathbb{R}_{+}$. Finally, we also find that

$$
\left(\mathcal{A} K_{p}\right)(x)-r K_{p}(x)+\pi(x)= \begin{cases}\beta^{-1}(\theta(x)-\theta(\hat{x})) & x \geq \hat{x} \\ 0 & x<\hat{x} .\end{cases}
$$

Since the state $\hat{x}$ is attained on the set where $\theta$ is strictly decreasing, we find that $\left(\mathcal{A} K_{p}\right)(x)-r K_{p}(x)+\pi(x) \leq 0$ for all $x \in \mathbb{R}_{+}$. Thus, the proposed value function $K_{p}(x)$ satisfies the conditions of Lemma 1 in 1 and, therefore, $K_{p}(x) \geq K(x)$ for all $x \in \mathbb{R}_{+}$.

Lemma 3.3 states a set of weak conditions under which the associated singular stochastic control problem (3.4) is solvable. A set of interesting comparative static results implied by Lemma 3.3 are now summarized in the following.

Corollary 3.4. Assume that the conditions of Lemma 3.3 are satisfied. Then

(i) the value $K(x)$ and the marginal value $K^{\prime}(x)$ of the optimal policy are decreasing functions of the parameter $\beta$;

(ii) the value $K(x)$ and the marginal value $K^{\prime}(x)$ of the optimal policy are increasing functions of the parameter $\lambda$;

(iii) the optimal exercise threshold $\hat{x}$ is an increasing mapping of the parameter $\beta$ and a decreasing mapping of the parameter $\lambda$.

Proof. (i) Denote the value associated with the parameter $\beta_{i}$ as $K_{i}(x), i=$ 1,2. It is now clear from the proof of Lemma 3.3 that $K_{2}(x)$ satisfies the sufficient variational inequalities $\left(\mathcal{A} K_{2}\right)(x)-r K_{2}(x)+\pi(x) \leq 0$ and $K_{2}^{\prime}(x) \geq$ $\lambda / \beta_{2}>\lambda / \beta_{1}$ for all $x \in \mathbb{R}_{+}$. Hence, $K_{2}(x) \geq K_{1}(x)$ for all $x \in \mathbb{R}_{+}$. In order to establish that $K_{2}^{\prime}(x) \geq K_{1}^{\prime}(x)$ we observe that the mapping $(\lambda-$ $\left.\beta\left(R_{r} \pi\right)^{\prime}(x)\right) /\left(\beta \psi^{\prime}(x)\right)$ is a decreasing function of the parameter $\beta$ from which the alleged result follows by invoking the representation (3.8). Proving part 
(ii) is entirely analogous. It remains to consider the sensitivity of the optimal exercise threshold $\hat{x}$ with respect to parametric changes. To this end, consider the mapping

$$
\bar{L}(x, \lambda, \beta)=r \int_{0}^{x} \psi(y)(\beta \pi(y)+\lambda \rho(y)) m^{\prime}(y) d y-(\beta \pi(x)+\lambda \rho(x)) \frac{\psi^{\prime}(x)}{S^{\prime}(x)} .
$$

If $\beta_{1}>\beta_{2}$ then

$$
\begin{aligned}
\bar{L}\left(x, \lambda, \beta_{1}\right)-\bar{L}\left(x, \lambda, \beta_{2}\right) & =\left(\beta_{1}-\beta_{2}\right)\left[r \int_{0}^{x} \psi(y) \pi(y) m^{\prime}(y) d y-\pi(x) \frac{\psi^{\prime}(x)}{S^{\prime}(x)}\right] \\
& \leq 0
\end{aligned}
$$

since

$$
r \int_{0}^{x} \psi(y) \pi(y) m^{\prime}(y) d y-\pi(x) \frac{\psi^{\prime}(x)}{S^{\prime}(x)} \leq-\pi(x) \frac{\psi^{\prime}(0)}{S^{\prime}(0)} \leq 0
$$

by the assumed monotonicity and non-negativity of $\pi(x)$. Therefore, if $\hat{x}_{i}$ denotes the optimal exercise threshold associated with $\beta_{i}, i=1,2$, we observe that $0=\bar{L}\left(\hat{x}_{1}, \lambda, \beta_{1}\right) \leq \bar{L}\left(\hat{x}_{1}, \lambda, \beta_{2}\right)$ which, in turn, implies that $\hat{x}_{1} \geq \hat{x}_{2}$. Establishing that $\hat{x}$ is a decreasing mapping of the parameter $\lambda$ is entirely analogous.

Corollary 3.4 characterizes the impact of parametric changes on the value, the marginal value and the optimal exercise threshold of the irreversible policy. We observe that an increase in $\beta$ decreases both the value and the marginal value of the optimal policy and, therefore, postpones exercise by increasing the optimal exercise threshold. The contrary happens when the parameter $\lambda$ increases. An interesting implication of these comparative static results is that parametric changes are neutral (i.e. do not affect the optimal exercise threshold $\hat{x})$ as long as the ratio $\lambda / \beta$ is held constant.

It is worth emphasizing that the value (3.7) of the optimal singular stochastic control can be re-expressed as

$$
K(x)= \begin{cases}\beta^{-1}(\lambda x+J(\hat{x})) & x \geq \hat{x} \\ \left(R_{r} \pi\right)(x)-\beta^{-1} I(\hat{x}) \psi(x) & x<\hat{x} .\end{cases}
$$

As we will notice in the subsequent analysis, (3.9) is closely related to the value of the considered impulse control problem. Moreover, as our next lemma indicates, the value (3.9) has an interesting maximality property summarized 
in the following lemma extending the results obtained in 7 in a model subject to a linear exercise payoff payoff.

Lemma 3.5. Define the continuously differentiable mapping $H: \mathbb{R}_{+}^{2} \rightarrow \mathbb{R}_{+}$as

$$
H(x, y)= \begin{cases}\beta^{-1}(\lambda x+J(y)) & x \geq y \\ \left(R_{r} \pi\right)(x)-\beta^{-1} I(y) \psi(x) & x<y\end{cases}
$$

and assume that the conditions of the Lemma 3.3 are met. Then $K(x)=$ $H(x, \hat{x})>H(x, y)$ and $K^{\prime}(x)=H_{x}(x, \hat{x})>H_{x}(x, y)$ for all $(x, y) \in \mathbb{R}_{+} \times$ $\mathbb{R} \backslash\{\hat{x}\}$. Moreover, $H_{y}(x, y)<0$, for all $(x, y) \in \mathbb{R}_{+} \times(\hat{x}, \infty)$.

Proof. Assume first, that $y>\hat{x}$. Then we find that

$$
H(x, \hat{x})-H(x, y)= \begin{cases}\beta^{-1}(J(\hat{x})-J(y)) & \hat{x}<y \leq x \\ \beta^{-1}(\lambda x+I(y) \psi(x)+J(\hat{x}))-\left(R_{r} \pi\right)(x) & \hat{x} \leq x<y \\ \beta^{-1} \psi(x)(I(y)-I(\hat{x})) & x<\hat{x}<y .\end{cases}
$$

We have shown earlier as a consequence of Lemma 3.1 that

$$
\hat{x}=\operatorname{argmin}\{I(x)\}=\operatorname{argmax}\{J(x)\} .
$$

Therefore, $I(y)-I(\hat{x})>0$ and $J(\hat{x})-J(y)>0$ for all $y \neq \hat{x}$. Consider now the difference $H(x, \hat{x})-H(x, y)$ on the interval $[\hat{x}, y)$. Since $J$ is monotonously decreasing on the interval $[\hat{x}, \infty)$, we find that

$$
\begin{aligned}
& \beta^{-1}(\lambda x+I(y) \psi(x)+J(\hat{x}))-\left(R_{r} \pi\right)(x) \\
\geq & \beta^{-1}(\lambda x+I(y) \psi(x)+J(x))-\left(R_{r} \pi\right)(x) \\
= & \beta^{-1} \psi(x)(I(y)-I(x))>0 .
\end{aligned}
$$

This observation proves that $H(x, \hat{x})>H(x, y)$ for all $x \in \mathbb{R}_{+}$whenever $y>\hat{x}$. In order to verify the second case assume now that $y<\hat{x}$. In that case

$$
H(x, \hat{x})-H(x, y)= \begin{cases}\beta^{-1}(J(\hat{x})-J(y)) & y<\hat{x} \leq x \\ \left(R_{r} \pi\right)(x)-\beta^{-1}(\lambda x-I(\hat{x}) \psi(x)-J(y)) & y \leq x<\hat{x} \\ \beta^{-1} \psi(x)(I(y)-I(\hat{x})) & x<y<\hat{x} .\end{cases}
$$


By relying on similar arguments as above we find that it suffices to consider the difference $H(x, \hat{x})-H(x, y)$ on the interval $[y, \hat{x})$. Again the monotonicity of $J$ implies that

$$
\begin{aligned}
& \left(R_{r} \pi\right)(x)-\beta^{-1}(\lambda x-I(\hat{x}) \psi(x)-J(y)) \\
\geq & \left(R_{r} \pi\right)(x)-\beta^{-1}(\lambda x-I(\hat{x}) \psi(x)-J(x)) \\
= & \beta^{-1} \psi(x)(I(x)-I(\hat{x}))>0 .
\end{aligned}
$$

Consequently, we find that $H(x, \hat{x})-H(x, y)>0$ for all $(x, y) \in \mathbb{R}_{+} \times \mathbb{R}_{+} \backslash$ $\{\hat{x}\}$. Establishing that $H_{x}(x, \hat{x})-H_{x}(x, y)>0$ for all $(x, y) \in \mathbb{R}_{+} \times \mathbb{R}_{+} \backslash\{\hat{x}\}$ is completely analogous. It remains yet to show that $H_{y}(x, y)<0$ for all $(x, y) \in \mathbb{R}_{+} \times(\hat{x}, \infty)$. Ordinary differentiation yields that

$$
H_{y}(x, y)= \begin{cases}\beta^{-1} J^{\prime}(y) & x \geq y \\ -\beta^{-1} \psi(x) I(y) & x<y\end{cases}
$$

This quantity is negative, since $J$ is monotonously decreasing on $(\hat{x}, \infty)$. This completes the proof of the lemma.

Lemma 3.5 shows that the value of the associated singular stochastic control problem does not only dominate but also grows faster than any other solution of the associated free boundary value problem (see 7 for a similar observation in a model subject to a linear exercise payoff)

$$
\begin{aligned}
& (\mathcal{A} u)(x)-r u(x)+\pi(x)=0, \quad x<y \\
& u^{\prime}(x)=\lambda / \beta, \quad x \geq y .
\end{aligned}
$$

This result is of interest since it emphasizes the role of the flexibility of the admissible policy as the main determinant of both the actual value and its growth rate. As we will later observe, it is these variational inequalities which relate the considered stochastic impulse control problem to both the associated singular stochastic control problem and to the associated optimal stopping problem.

\subsection{THE ASSOCIATED OPTIMAL STOPPING PROBLEM}

Let $X_{t}$ be the diffusion evolving on $\mathbb{R}_{+}$according to the ordinary Itô stochastic differential equation (2.2) and assume that the infinitesimal coefficients $\mu$ and 
$\sigma$ satisfy the same regularity conditions as in the impulse control case. Given these assumptions, consider the corresponding optimal stopping problem

$$
G_{c}(x)=\sup _{\tau<\tau_{0}} \mathbf{E}_{x}\left[\int_{0}^{\tau} e^{-r s} \pi\left(X_{s}\right) d s+e^{-r \tau}\left(\lambda \beta^{-1} X_{\tau}-c\right)\right],
$$

where $c \geq 0$ is an arbitrary constant and $\tau$ is an arbitrary $\mathcal{F}_{t}$-stopping time satisfying the constraint $\tau<\tau_{0}$ stating that the stopping time problem is defined up to the first date the underlying diffusion exits from its state-space $\mathbb{R}_{+}$. Along the lines indicated by (3.5) we find by applying Dynkin's theorem to the mapping $x \mapsto \lambda x / \beta-c$ that

$$
G_{c}(x)=\frac{\lambda}{\beta} x-c+\frac{1}{\beta} \sup _{\tau<\tau_{0}} \mathbf{E}_{x} \int_{0}^{\tau} e^{-r s}\left(\theta\left(X_{s}\right)+\beta c r\right) d s
$$

demonstrating how the value of the optimal policy can in this case be decomposed into the sum of the immediate exercise payoff and the early exercise premium. The next lemma will present a substantially deep relationship between the associated singular control and the associated optimal stopping problem.

Lemma 3.6. Assume, that the conditions of Lemma 3.3 are met and that $c \geq 0$. Then $K(x)>G_{c}(x)$ and $K^{\prime}(x)>G_{c}^{\prime}(x)$, where

$$
\begin{aligned}
G_{c}(x) & =\left(R_{r} \pi\right)(x)+\beta^{-1} \psi(x) \sup _{y \geq x}\left[\frac{\lambda y-\beta\left(R_{r} \pi\right)(y)-\beta c}{\psi(y)}\right] \\
& = \begin{cases}\beta^{-1} \lambda x-c & x \geq \bar{x}_{c} \\
\left(R_{r} \pi\right)(x)-\beta^{-1} I\left(\bar{x}_{c}\right) \psi(x) & x<\bar{x}_{c}\end{cases}
\end{aligned}
$$

denotes the value of the optimal stopping problem and $\bar{x}_{c}$, denoting the optimal stopping threshold, is the unique root of the equation $J\left(\bar{x}_{c}\right)=-\beta c$.

Proof. In order to establish (3.11), denote as $x_{0}$ the unique interior state at which $\theta\left(x_{0}\right)=0$. Since

$$
\frac{\psi^{\prime}(x)}{S^{\prime}(x)} J(x)=\int_{0}^{x} \psi(y) \theta(y) m^{\prime}(y) d y,
$$

we find that

$$
\frac{d}{d x}\left[\frac{\psi^{\prime}(x)}{S^{\prime}(x)} J(x)\right]=\psi(x) \theta(x) m^{\prime}(x) \gtreqless 0, x \lesseqgtr x_{0} .
$$


Since $\lim _{x \downarrow 0} \frac{\psi^{\prime}(x)}{S^{\prime}(x)} J(x) \geq 0$, we consequently discover that $J(x)>0$ whenever $x \in\left(0, x_{0}\right)$. On the other hand, we proved in the Lemma 3.2 that $\lim _{x \rightarrow \infty} J(x)=-\infty$. Together with the monotonicity properties of $J$, this demonstrates that there exists an unique state $\bar{x}_{c} \in \theta^{-1}\left(\mathbb{R}_{-}\right)$at which the condition $J\left(\bar{x}_{c}\right)=-\beta c$ is satisfied. Moreover, ordinary differentiation yields

$$
\frac{d}{d x}\left[\frac{\lambda x-\beta\left(R_{r} \pi\right)(x)-\beta c}{\psi(x)}\right]=\frac{\psi^{\prime}(x)}{\psi^{2}(x)}(J(x)+\beta c),
$$

which in turn implies that

$$
\bar{x}_{c}=\operatorname{argmax}\left\{\frac{\lambda x-\beta\left(R_{r} \pi\right)(x)-\beta c}{\psi(x)}\right\} .
$$

Given these observations, denote the proposed value function as $\hat{G}_{c}(x)$. Since

$$
\hat{G}_{c}(x)=\mathbf{E}_{x}\left[\int_{0}^{\bar{\tau}} e^{-r s} \pi\left(X_{s}\right) d s+e^{-r \bar{\tau}}\left(\lambda \beta^{-1} X_{\bar{\tau}}-c\right)\right],
$$

where $\bar{\tau}=\inf \left\{t \geq 0 \mid X_{t} \geq \bar{x}_{c}\right\}$, we find that $\hat{G}_{c}(x) \leq G_{c}(x)$. On the other hand, we also observe that the proposed value function $\hat{G}_{c}(x)$ is continuously differentiable on $\mathbb{R}_{+}$, twice continuously differentiable on $\mathbb{R}_{+} \backslash\left\{\bar{x}_{c}\right\}$ and satisfies the variational inequality $\min \left\{r \hat{G}_{c}(x)-\left(\mathcal{A} \hat{G}_{c}\right)(x)-\pi(x), \hat{G}_{c}(x)-\right.$ $\left.\lambda \beta^{-1} x+c\right\}=0$. Thus, $\hat{G}_{c}(x) \geq G_{c}(x)$, which finally implies that $\hat{G}_{c}(x)=$ $G_{c}(x)$.

In order to prove that $K(x) \geq G_{c}(x)$, observe that the value of the associated singular control problem satisfies the variational inequality $(\mathcal{A} K)(x)-$ $r K(x)+\pi(x) \leq 0$ and the inequality

$$
K(x)-\lambda \beta^{-1} x+c \geq \beta^{-1} J(\min (x, \hat{x})) \geq 0 .
$$

Thus, $K(x)$ satisfies the sufficient variational inequalities guaranteeing that $K(x) \geq G_{c}(x)$. The inequality $K^{\prime}(x) \geq G_{c}^{\prime}(x)$ is now a straightforward consequence of (3.8) and Lemma 3.5

Lemma 3.6 states a set of conditions under which the associated optimal stopping problem is solvable. Interestingly, we find that both the value and the marginal value of the optimal timing policy are smaller than the value and the marginal value of the associated singular stochastic control problem, respectively. A set of interesting comparative static results implied by Lemma 3.6 are now summarized in the following. 
Corollary 3.7. Assume that the conditions of Lemma 3.6 are satisfied. Then

(i) the value $G_{c}(x)$ is a decreasing function of both the parameter $\beta$ and the sunk cost $c$ and an increasing function of the parameter $\lambda$;

(ii) the optimal exercise threshold $\bar{x}_{c}$ is an increasing mapping of both the parameter $\beta$ and the sunk cost $c$ and a decreasing mapping of the parameter $\lambda$.

Proof. The claim of part (i) of our corollary follow directly from the definition of the exercise payoff. Thus, it is sufficient to consider the sensitivity of the optimal threshold $\bar{x}_{c}$ to changes in either $\lambda, \beta$, or $c$. To this end, consider the mapping

$$
\tilde{L}(x, \lambda, \beta, c)=\int_{0}^{x} \psi(y)(\beta \pi(y)+\lambda \rho(y)) m^{\prime}(y) d y+\beta c \frac{\psi^{\prime}(x)}{S^{\prime}(x)}
$$

and denote as $\bar{x}_{c}\left(\beta_{i}\right)$ the optimal exercise threshold associated with the parameter $\beta_{i}$. If $\beta_{1}>\beta_{2}$ then

$$
\begin{aligned}
\tilde{L}\left(x, \lambda, \beta_{1}, c\right)-\tilde{L}\left(x, \lambda, \beta_{2}, c\right) & =\left(\beta_{1}-\beta_{2}\right)\left[\int_{0}^{x} \psi(y) \pi(y) m^{\prime}(y) d y+c \frac{\psi^{\prime}(x)}{S^{\prime}(x)}\right] \\
& >0
\end{aligned}
$$

which implies that $0=\tilde{L}\left(\bar{x}_{c}\left(\beta_{1}\right), \lambda, \beta_{1}, c\right)>\tilde{L}\left(\bar{x}_{c}\left(\beta_{1}\right), \lambda, \beta_{2}, c\right)$ and, therefore, that $\bar{x}_{c}\left(\beta_{1}\right)>\bar{x}_{c}\left(\beta_{2}\right)$. The analysis of the impact of changes in either $\lambda$ or $c$ on the optimal exercise threshold is entirely analogous.

Corollary 3.7 extends the findings of Corollary 3.4 to the present example. More precisely, we observe that an increases in $\beta$ postpones rational exercise by expanding the continuation region where stopping is suboptimal. The opposite is shown to happen when $\lambda$ increases. Interestingly, we again find that parametric changes are neutral (i.e. do not affect the optimal exercise threshold $\bar{x}_{c}$ ) as long as the ratio $\lambda / \beta$ is held constant. Moreover, as intuitively is clear, our findings indicate that increased sunk costs decrease the value and postpone rational exercise by expanding the continuation region. 


\section{OPTIMAL IMPULSE CONTROL POLICY}

\subsection{NECESSARY CONDITIONS}

The stochastic impulse control problems of type (2.4) are typically tackled by relying on either dynamic programming techniques or on quasi-variational inequalities. In this study, we plan to adopt an alternative approach which yields more tangible and easily interpretable conditions on the optimality of the control policy. Instead of considering the class of all admissible impulse controls at once, we restrict our attention to the subclass $\left\{\nu_{(\zeta, y)}\right\}$ of admissible impulse controls characterized by the sequence of intervention times $\tau_{0}^{y}=$ $0, \tau_{k}^{y}=\inf \left\{t \geq \tau_{k-1}^{y} \mid X_{t}^{\nu} \geq y\right\}$ and the sequence of interventions $\zeta_{k}^{y}=$ $\zeta+(x-y)^{+}$, for all $k \geq 1$. That is, we restrict our attention interest to control policies consisting of sequence of constant-sized impulses (with the exception of the initial impulse which depends on the initial state) exerted every time the underlying diffusion hits a predetermined, constant exercise threshold $y$. Given this class of admissible impulse controls, define the value $F_{c}: \mathbb{R}_{+} \rightarrow \mathbb{R}$ accrued from applying the impulse control $\nu_{(\zeta, y)}$ as $F_{c}(x)=J_{c}^{(\zeta, y)}(x)$. Since $X_{\tau_{k}+}=X_{\tau_{k^{-}}}-\beta \zeta$ for all $k$ and the controlled diffusion evolves as the linear diffusion $X_{t}$ between any two successive intervention dates, we observe that for all $x<y$, the value satisfies the functional relation (a so-called running present value formulation)

$F_{c}(x)=\mathbf{E}_{x}\left[\int_{0}^{\tau_{y}} e^{-r s} \pi\left(X_{s}\right) d s+e^{-r \tau_{y}}\left(\lambda\left(X_{\tau_{y}}-(y-\zeta)\right)-c+F_{c}(y-\beta \zeta)\right)\right]$,

where $\tau_{y}=\inf \left\{t \geq 0 \mid X_{t} \geq y\right\}$. Invoking the strong Markov property of diffusions now implies that the value $F_{c}(x)$ can be represented as

$$
F_{c}(x)= \begin{cases}F_{c}(y-\beta \zeta)+\lambda(x-y+\zeta)-c & x \geq y \\ \left(R_{r} \pi\right)(x)+\left(\lambda \zeta-c-\left(R_{r} \pi\right)(y)+F_{c}(y-\beta \zeta)\right) \frac{\psi(x)}{\psi(y)} & x<y\end{cases}
$$

First of all, note that letting $x$ tend to $y$ in (4.2) yields the value-matching condition $F_{c}(y)=F_{c}(y-\beta \zeta)+\lambda \zeta-c$ which can be re-expressed in the more familiar form $F_{c}(y-\beta \zeta)+\lambda \zeta=F_{c}(y)+c$ stating that the value of the investment opportunity has to coincide with its full costs (lost option value + 
sunk cost). On the other hand, by letting $x$ tend to $y-\beta \zeta$ yields

$$
F_{c}(y-\beta \zeta)=\frac{\psi(y)\left(R_{r} \pi\right)(y-\beta \zeta)+\left[\lambda \zeta-c-\left(R_{r} \pi\right)(y)\right] \psi(y-\beta \zeta)}{\psi(y)-\psi(y-\beta \zeta)} .
$$

Now, inserting (4.3) into (4.2) implies that that the value can be expressed as

$$
F_{c}(x)= \begin{cases}\left(R_{r} \pi\right)(y-\beta \zeta)+h(\zeta, y) \psi(y-\beta \zeta)+\lambda(x-y+\zeta)-c & x \geq y \\ \left(R_{r} \pi\right)(x)+h(\zeta, y) \psi(x) & x<y\end{cases}
$$

where the mapping $h: \mathbb{R}_{+}^{2} \rightarrow \mathbb{R}$ is defined as

$$
h(\zeta, y)=\frac{\left(R_{r} \pi\right)(y-\beta \zeta)-\left(R_{r} \pi\right)(y)+\lambda \zeta-c}{\psi(y)-\psi(y-\beta \zeta)} .
$$

In order to prove the existence and uniqueness of the optimal impulse control policy, we will consider the ordinary inequality constrained non-linear programming problem

$$
\sup _{\substack{\beta \zeta \in[0, y], y \in \mathbb{R}_{+}}} \frac{\left(R_{r} \pi\right)(y-\beta \zeta)-\left(R_{r} \pi\right)(y)+\lambda \zeta-c}{\psi(y)-\psi(y-\beta \zeta)} .
$$

To ease up the subsequent analysis, introduce a linear change of variables: let $z=y-\beta \zeta$. Thus $\zeta=\beta^{-1}(y-z)$. Since the parameter $\beta$ is assumed to be positive, the programming problem (4.6) can now be re-written as

$$
\sup _{\substack{z \in[0, y] \\ y \in \mathbb{R}_{+}}} \frac{\left(R_{r} \pi\right)(z)-\left(R_{r} \pi\right)(y)+\lambda \beta^{-1}(y-z)-c}{\psi(y)-\psi(z)} .
$$

If an interior pair maximizing the mapping $h$ and, therefore, satisfying the problem (4.7) exists, denote the value associated to this pair as $F_{c}^{*}(x)$. To express the same in a more accurate manner, if an interior pair $\left(z_{c}^{*}, y_{c}^{*}\right)$ satisfying the problem (4.7) exists, define the mapping $F_{c}^{*}: \mathbb{R} \rightarrow \mathbb{R}$ as

$$
F_{c}^{*}(x)= \begin{cases}\left(R_{r} \pi\right)\left(z_{c}^{*}\right)+h\left(z_{c}^{*}, y_{c}^{*}\right) \psi\left(z_{c}^{*}\right)+l\left(x, y_{c}^{*}, z_{c}^{*}\right)-c & x \geq y_{c}^{*} \\ \left(R_{r} \pi\right)(x)+h\left(z_{c}^{*}, y_{c}^{*}\right) \psi(x) & x<y_{c}^{*},\end{cases}
$$

where $l(x, y, z)=\lambda\left(x-y-\beta^{-1}(y-z)\right)$. Since $h$ is a differentiable function, it is clear that if an interior pair $\left(z_{c}^{*}, y_{c}^{*}\right)$ satisfying the problem (4.7) exists, 
then this pair satisfies the ordinary necessary first-order conditions $\frac{\partial h}{\partial z}\left(z_{c}^{*}, y_{c}^{*}\right)=$ $\frac{\partial h}{\partial y}\left(z_{c}^{*}, y_{c}^{*}\right)=0$. More precisely, if a an optimal pair exists, it must satisfy the conditions

$$
\left\{\begin{array}{l}
\left(\psi\left(y_{c}^{*}\right)-\psi\left(z_{c}^{*}\right)\right)\left(\lambda \beta^{-1}-\left(R_{r} \pi\right)^{\prime}\left(y_{c}^{*}\right)\right)=r\left(z_{c}^{*}, y_{c}^{*}\right) \psi^{\prime}\left(y_{c}^{*}\right) \\
\left(\psi\left(y_{c}^{*}\right)-\psi\left(z_{c}^{*}\right)\right)\left(\lambda \beta^{-1}-\left(R_{r} \pi\right)^{\prime}\left(z_{c}^{*}\right)\right)=r\left(z_{c}^{*}, y_{c}^{*}\right) \psi^{\prime}\left(z_{c}^{*}\right),
\end{array}\right.
$$

where $r(z, y)=\left(R_{r} \pi\right)(z)-\left(R_{r} \pi\right)(y)+\lambda \beta^{-1}(y-z)-c$. This yields immediately the condition

$$
\frac{\lambda-\beta\left(R_{r} \pi\right)^{\prime}\left(z_{c}^{*}\right)}{\psi^{\prime}\left(z_{c}^{*}\right)}=\frac{\lambda-\beta\left(R_{r} \pi\right)^{\prime}\left(y_{c}^{*}\right)}{\psi^{\prime}\left(y_{c}^{*}\right)} .
$$

Using the notation introduced in the subsection 3.1, this can be rewritten as

$$
I\left(y_{c}^{*}\right)-I\left(z_{c}^{*}\right)=0 .
$$

On the other hand, since

$$
\frac{\psi^{\prime}\left(z_{c}^{*}\right)}{\psi\left(y_{c}^{*}\right)-\psi\left(z_{c}^{*}\right)}=\frac{\left(R_{r} \pi\right)^{\prime}\left(z_{c}^{*}\right)-\lambda \beta^{-1}}{r\left(z_{c}^{*}, y_{c}^{*}\right)},
$$

we find by invoking condition (4.10), reordering terms that

$\left[\beta\left(R_{r} \pi\right)\left(y_{c}^{*}\right)-I\left(y_{c}^{*}\right) \psi\left(y_{c}^{*}\right)-\lambda y_{c}^{*}\right]-\left[\beta\left(R_{r} \pi\right)\left(z_{c}^{*}\right)-I\left(z_{c}^{*}\right) \psi\left(z_{c}^{*}\right)-\lambda z_{c}^{*}\right]=-\beta c$.

Again, with the notation from the subsection 3.1, this can be expressed as

$$
J\left(y_{c}^{*}\right)-J\left(z_{c}^{*}\right)=-\beta c .
$$

The conditions (4.10) and (4.11) are standard necessary first-order conditions for the existence of the solution of the problem (4.7). In the next subsection, we will use the results derived in the subsection 3.1 in order to prove that under the assumptions Lemma 3.1 there indeed exists a unique interior pair $\left(z_{c}^{*}, y_{c}^{*}\right)$ satisfying the algebraic equations (4.10) and (4.11). This pair then induces an unique solution $\left(\zeta_{c}^{*}, y_{c}^{*}\right)$ for the non-linear programming problem (4.6).

\subsection{EXISTENCE AND SUFFICIENCY}

Having presented a set of necessary conditions for the optimality of the considered subclass of admissible stochastic impulse controls, we now establish a set of weak conditions under which the necessary conditions (4.10) and (4.11) have indeed a unique solution. 
Lemma 4.1. Assume that the conditions of Lemma 3.1 are met. Then there exists an unique interior pair $\left(z_{c}^{*}, y_{c}^{*}\right)$ for which the necessary conditions (4.10) and (4.11) are satisfied.

Proof. Existence. Define the mappings $\check{J}:(0, \hat{x}] \rightarrow(\breve{J}(0), \breve{J}(\hat{x})]$ and $\hat{J}$ : $(\hat{x}, \infty) \rightarrow(-\infty, \hat{J}(\hat{x}))$ as restrictions of the mapping $J$ and the mapping $k$ : $\mathbb{R} \rightarrow \mathbb{R}$ as $k(x)=x-\beta c$. By virtue of the properties of $J$, it is clear that both of the mappings $\breve{J}$ and $\hat{J}$ are continuous and bijective. Now, define the mapping $\hat{y}:(0, \hat{x}) \rightarrow(\hat{y}(\hat{x}), \hat{y}(0))$ as $\hat{y}(x)=\left(\breve{J} \circ k \circ \hat{J}^{-1}\right)(x)$. By the definitions of $\breve{J}, \hat{J}$ and $k$, we observe that $\hat{y}$ is well-defined. Moreover, the mapping $\hat{y}$ is continuous and bijective as a composition of continuous and bijective mappings. Finally, since $\hat{y}(z)=\hat{J}^{-1}(\breve{J}(z)-\beta c)$, we find that the equation $J(\hat{y}(z))-J(z)=-\beta c$ holds for all $z \in(0, \hat{x}]$.

Analogously to the notation used above, define the mappings $\check{I}:(0, \hat{x}] \rightarrow$ $[\check{I}(\hat{x}), \check{I}(0))$ and $\hat{I}:(\hat{x}, \infty) \rightarrow(\hat{I}(\hat{x}), \hat{I}(0))$ as restrictions of the mapping $I$. Define the mapping $Y:(\breve{I}(\hat{x}), \check{I}(0)) \rightarrow(\hat{I}(\hat{y}(\hat{x})), \hat{I}(\hat{y}(0)))$ as $Y(x)=\left(\check{I}^{-1} \circ\right.$ $\hat{y} \circ \hat{I})(x)$. Since $0<\beta c<\infty$ and $\hat{J}$ is decreasing, we discover that $\hat{y}(\hat{x})=$ $\hat{J}^{-1}(\breve{J}(\hat{x})-\beta c)>\hat{J}^{-1}(\breve{J}(\hat{x}))=\hat{x}$ and $\hat{y}(0)=\hat{J}^{-1}(\breve{J}(0)-\beta c) \leq \hat{J}^{-1}(-\beta c)<$ $\infty$. Firstly, these findings guarantee that $Y$ is well-defined. Moreover, these same findings coupled with Lemma 3.2 imply that

$$
\left.(\hat{I}(\hat{y}(\hat{x})), \hat{I}(\hat{y}(0))) \subsetneq(\hat{I}(\hat{x}), \hat{I}(x))\right|_{x=\infty} \subseteq(\check{I}(\hat{x}), 0) \subseteq(\check{I}(\hat{x}), \check{I}(0)) .
$$

In other words, we discover that the image of $Y$ is strictly included in the domain of $Y$. This observation coupled with the fact that $Y$ is continuous and bijective leads us to the conclusion that the mapping $Y$ has a fixed point. In other words, there exists a state $z^{*} \in(0, \hat{x})$, for which $I\left(z^{*}\right)=\check{I}\left(z^{*}\right)=$ $\hat{I}\left(\hat{y}\left(z^{*}\right)\right)=I\left(\hat{y}\left(z^{*}\right)\right)$. Moreover, since $z^{*} \in(0, \hat{x}]$, the equation $J\left(\hat{y}\left(z^{*}\right)\right)-$ $J\left(z^{*}\right)=-\beta c$ also holds. These observations complete the first part of the proof.

Uniqueness. Assume, that $\left(z^{*}, \hat{y}\left(z^{*}\right)\right)$ is a solution for the conditions (4.10) and (4.11). Due to the Lemma 3.2, we know that there exist such a state $\check{x} \in(0, \hat{x})$ that $I(x)<0$ for all $x \in(\check{x}, \infty)$. By virtue of this observation, define the mapping $\check{y}:(\check{x}, \hat{x}) \rightarrow(\hat{x}, \infty)$ as $\hat{y}(x)=\left(\check{I} \circ \hat{I}^{-1}\right)(x)$. Using the same arguments as with the mapping $\hat{y}$, we find that $\check{y}$ is well-defined, continuous, bijective and that the equation $I(\check{y}(z))-I(z)=0$ holds for all $z \in(\check{x}, \hat{x})$. 
Consider now the slopes of the implicit curves $I(\check{y}(z))-I(z)=0$ and $J(\hat{y}(z))-J(z)=-\beta c$ at the point $\left(z^{*}, \hat{y}\left(z^{*}\right)\right)$. Standard differentiation yields that

$$
\hat{y}^{\prime}\left(z^{*}\right)=\frac{J^{\prime}\left(z^{*}\right)}{J^{\prime}\left(\hat{y}\left(z^{*}\right)\right)}=\frac{\psi\left(z^{*}\right)}{\psi\left(\hat{y}\left(z^{*}\right)\right)} \frac{I^{\prime}\left(z^{*}\right)}{I^{\prime}\left(\hat{y}\left(z^{*}\right)\right)}>\frac{I^{\prime}\left(z^{*}\right)}{I^{\prime}\left(\check{y}\left(z^{*}\right)\right)}=\check{y}^{\prime}\left(z^{*}\right),
$$

since by assumption $\left(z^{*}, \hat{y}\left(z^{*}\right)\right)=\left(z^{*}, \check{y}\left(z^{*}\right)\right)$. This computation proves the uniqueness of the solution.

Lemma 4.1 demonstrates that the conditions of Lemma 3.1 are sufficient for both the existence and uniqueness of a solution for the typically highly nonlinear necessary conditions (4.10) and (4.11). It is worth pointing out that since the existence result of Lemma 4.1 is based on a fixed point argument, the existence of a potentially optimal pair is guaranteed for a considerably broad class of problems. Given the results of Lemma 4.1, we are now in position to state our main result on the optimal stochastic impulse control policy and its value.

Theorem 4.2. Assume, that the conditions of Lemma 3.1 are satisfied. Then, the unique optimal impulse control policy is $\nu^{*}=\nu_{\left(\zeta_{c}^{*}, y_{c}^{*}\right)}$. Moreover, the value of the optimal impulse control policy reads as

$$
V_{c}(x)=F_{c}^{*}(x)= \begin{cases}\beta^{-1}\left(\lambda x+J\left(y_{c}^{*}\right)\right) & x \geq y_{c}^{*} \\ \left(R_{r} \pi\right)(x)-\beta^{-1} I\left(y_{c}^{*}\right) \psi(x) & x<y_{c}^{*} .\end{cases}
$$

Proof. Denote the proposed value function as $V_{c}^{p}(x)$. Since the policy described above is admissible, it is clear that $V_{c}^{p}(x) \leq V_{c}(x)$. To prove the opposite, we first observe that $V_{c}^{p} \in C^{1}\left(\mathbb{R}_{+}\right) \cap C^{2}\left(\mathbb{R}_{+} \backslash\left\{y_{c}^{*}\right\}\right)$ and that $V_{c}^{p^{\prime \prime}}\left(y_{c}^{*}+\right)=$ $0 \leq\left|\left(R_{r} \pi\right)^{\prime \prime}\left(y_{c}^{*}\right)-\beta^{-1} I\left(y_{c}^{*}\right) \psi^{\prime \prime}\left(y_{c}^{*}\right)\right|=\left|V_{c}^{p^{\prime \prime}}\left(y_{c}^{*}-\right)\right|<\infty$, meaning that the proposed value function $V_{c}^{p}$ is stochastically $C^{2}\left(\mathbb{R}_{+}\right)$. Moreover, by $r$ harmonicity of $\psi$ we have that $\left((\mathcal{A}-r) V_{c}^{p}\right)(x)+\pi(x)=0$ on $\left(0, y_{c}^{*}\right)$. For $x \in\left(y_{c}^{*}, \infty\right)$, we have $\left((\mathcal{A}-r) V_{c}^{p}\right)(x)+\pi(x)=\theta(x)-r \beta^{-1} J\left(y_{c}^{*}\right)$. However, since

$$
I^{\prime}(x)=\frac{2}{\sigma^{2}(x) \psi^{\prime}(x)}[r J(x)-\theta(x)]
$$

and $I(x)$ is non-decreasing on $\left(y_{c}^{*}, \infty\right)$, we find that $\theta(x) \leq r \beta^{-1} J(x)$ on $\left(y_{c}^{*}, \infty\right)$. Consequently, we find that for all $x \in\left(y_{c}^{*}, \infty\right)$ we have that $((\mathcal{A}-$ 
$\left.r) V_{c}^{p}\right)(x)+\pi(x) \leq r \beta^{-1}\left(J(x)-J\left(y_{c}^{*}\right)\right) \leq 0$, since $J(x)$ in non-increasing on $\left(y_{c}^{*}, \infty\right)$. Hence $\left((\mathcal{A}-r) V_{c}^{p}\right)(x)+\pi(x) \leq 0$ for all $x \in \mathbb{R}_{+} \backslash\left\{y_{c}^{*}\right\}$.

Our next task is to show that the proposed value $V_{c}^{p}(x)$ satisfies the quasivariational inequality

$$
V_{c}^{p}(x) \geq \sup _{\beta \zeta \in[0, x]}\left[V_{c}^{p}(x-\beta \zeta)+\lambda \zeta-c\right]
$$

for all $x \in \mathbb{R}_{+}$. Note, that this quasi-variational inequality can be written as well in the form

$$
V_{c}^{p}(x) \geq \beta^{-1}(\lambda x-\beta c)+\sup _{y \in[0, x]}\left[V_{c}^{p}(y)-\lambda \beta^{-1} y\right] .
$$

Define now the mapping $A: \mathbb{R}_{+} \rightarrow \mathbb{R}$ as

$$
A(x)=V_{c}^{p}(x)-\beta^{-1}(\lambda x-\beta c)-\sup _{y \in[0, x]}\left[V_{c}^{p}(y)-\lambda \beta^{-1} y\right] .
$$

Since

$$
V_{c}^{p^{\prime}}(x)= \begin{cases}\lambda \beta^{-1} & x \geq y_{c}^{*} \\ \beta^{-1}\left(\lambda+\psi^{\prime}(x)\left(I(x)-I\left(y_{c}^{*}-\beta \zeta_{c}^{*}\right)\right)\right) & x<y_{c}^{*}\end{cases}
$$

and $I(x)$ is decreasing on $\left(0, y_{c}^{*}\right)$, we observe that

$$
\sup _{y \in[0, x]}\left[V_{c}^{p}(y)-\lambda \beta^{-1} y\right]= \begin{cases}V_{c}^{p}\left(y_{c}^{*}-\beta \zeta_{c}^{*}\right)-\lambda \beta^{-1}\left(y_{c}^{*}-\beta \zeta_{c}^{*}\right) & x>y_{c}^{*}-\beta \zeta_{c}^{*} \\ V_{c}^{p}(x)-\lambda \beta^{-1} x & x \leq y_{c}^{*}-\beta \zeta_{c}^{*} .\end{cases}
$$

This implies that

$$
A(x)= \begin{cases}0 & x \geq y_{c}^{*} \\ \left(R_{r} \pi\right)(x)-I\left(y_{c}^{*}\right) \psi(x)-\lambda \beta^{-1} x-J\left(y_{c}^{*}\right) & x \in\left(y_{c}^{*}-\beta \zeta_{c}^{*}, y_{c}^{*}\right) \\ c & x \leq y_{c}^{*}-\beta \zeta_{c}^{*} .\end{cases}
$$

Since $\lim _{x \rightarrow y_{c}^{*}-} A(x)=0$ and $A^{\prime}(x)=\left(R_{r} \pi\right)(x)-I\left(y_{c}^{*}\right) \psi^{\prime}(x)-\lambda \beta^{-1}=$ $\psi^{\prime}(x)\left(I(x)-I\left(y_{c}^{*}\right)\right)<0$, we find that $A(x) \geq 0$ on $\left(y_{c}^{*}-\beta \zeta_{c}^{*}, y_{c}^{*}\right)$; hence $A(x) \geq 0$ for all $x \in \mathbb{R}$.

Finally, given the continuity of the proposed value function and the fact that the state-space $\left(0, y_{c}^{*}\right)$ of the controlled diffusion $X_{t}^{\nu}$ is bounded, we observe that $\mathbf{E}_{x}\left[e^{-r t} V_{c}^{p}\left(X_{t}^{\nu}\right)\right] \downarrow 0$ for all $x \in \mathbb{R}_{+}$as $t \rightarrow \infty$. Thus $V_{p}^{*}(x) \geq V_{c}^{p}(x)$ and $\nu^{*}=\nu_{\left(\zeta_{c}^{*}, y_{c}^{*}\right)}$. 
Theorem 4.2 demonstrates that the admissible policy $\nu^{*}=\nu_{\left(\zeta_{c}^{*}, y_{c}^{*}\right)}$ is indeed optimal and $F_{c}^{*}(x)$ is the value of the optimal policy whenever the conditions of Lemma 3.1 are satisfied. This observation is of interest since it emphasizes the role of the mapping $\theta(x)$ as the principal determinant of both the existence and uniqueness of an optimal policy. It is worth noticing that the conditions are considerably weak since no concavity assumptions are required and only the monotonicity and continuity properties of the mapping $\theta(x)$ are required for guaranteeing the validity of the results of Theorem 4.2. Our main characterization of the impact of the flexibility of the applied policy on the values and the marginal values of the considered stochastic control problems are now summarized in the following (cf. 7 for a similar observation in the linear payoff case).

Corollary 4.3. Assume, that the conditions of the Lemma 4.1 are met. Then

$$
K(x) \geq V_{c}(x) \geq G_{c}(x) \quad \text { and } \quad K^{\prime}(x) \geq V_{c}^{\prime}(x) \geq G_{c}^{\prime}(x)
$$

for all $x \in \mathbb{R}_{+}$. Moreover, $\bar{x}_{c}>y_{c}^{*}>\hat{x}$ for all $c>0$.

Proof. Inequality $K(x) \geq V_{c}(x)$ follows directly from Lemma 3.5 and the representation (4.12). On the other hand, as was established in the proof of Lemma 4.2, the value function $V_{c}(x)$ is continuously differentiable on whole of $\mathbb{R}_{+}$, twice continuously differentiable on $\mathbb{R}_{+} \backslash\left\{y_{c}^{*}\right\}$ and satisfies the variational inequality $\left(\mathcal{A} V_{c}\right)(x)-r V_{c}(x)+\pi(x) \leq 0$ for all $x \in \mathbb{R}_{+} \backslash\left\{y_{c}^{*}\right\}$. Moreover, since

$$
V_{c}(x) \geq \sup _{\beta \zeta \leq x}\left[\lambda \zeta-c+V_{c}(x-\beta \zeta)\right] \geq \lambda \beta^{-1} x-c,
$$

we observe that $V_{c}(x)$ satisfies the sufficient variational inequalities guaranteeing that $V_{c}(x) \geq G_{c}(x)$.

It is clear from the proof of the Lemma 4.1, that $y_{c}^{*}>\hat{x}$. Moreover, since

$$
0 \leq V_{c}(x)-G_{c}(x)=\beta^{-1} \psi(x)\left(I\left(\bar{x}_{c}\right)-I\left(y_{c}^{*}\right)\right)
$$

for all $x \in\left(0, \min \left(y_{c}^{*}, \bar{x}_{c}\right)\right)$ and both of the thresholds $\bar{x}_{c}$ and $y^{*}$ are attained on the set where $I(x)$ is non-decreasing, we discover that $\bar{x}_{c} \geq y_{c}^{*}$.

It remains to establish that $K^{\prime}(x) \geq V_{c}^{\prime}(x) \geq G_{c}^{\prime}(x)$. Again, the inequality $K^{\prime}(x) \geq V_{c}^{\prime}(x)$ follows directly from Lemma 3.5. Since $\bar{x}_{c} \geq y_{c}^{*} \geq \hat{x}$, we find that

$$
V_{c}^{\prime}(x)-G_{c}^{\prime}(x) \geq \begin{cases}\beta^{-1} J\left(y_{c}^{*}-\beta \zeta_{c}^{*}\right) & y_{c}^{*}<\bar{x}_{c} \leq x \\ \beta^{-1} \psi(x)\left[I\left(\bar{x}_{c}\right)-I(x)\right] & y_{c}^{*} \leq x<\bar{x}_{c} \text { or } x<y_{c}^{*}<\bar{x}_{c} .\end{cases}
$$


Consequently, we find that $V_{c}^{\prime}(x)-G_{c}^{\prime}(x) \geq 0$ since both of the thresholds $y_{c}^{*}$ and $\bar{x}_{c}$ are attained on the set where $I(x)$ is non-decreasing.

Corollary 4.3 extends the results of Lemma 3.6 to the present case. As intuitively is clear, we find that increased flexibility increases the value of the optimal decision. Interestingly, Corollary 4.3 also demonstrates that increased flexibility increases the marginal value of the optimal policy as well. Hence, we observe that increased flexibility does not only increase the values of optimal policies, it also increases the rate at which these values are increasing.

Having studied the existence and uniqueness of an optimal impulse control policy, we now plan to analyze the comparative static properties of the optimal policy and its value. In accordance with our earlier findings in Corollary 3.4 and Corollary 3.7 we can now establish the following

Corollary 4.4. Assume that the conditions of Lemma 3.1 are satisfied. Then the value $V_{c}(x)$ is a decreasing function of both the parameter $\beta$ and the sunk cost $c$ and an increasing function of the parameter $\lambda$.

Proof. Denote as $V_{c, \lambda_{i}}(x)$ the value of the optimal policy associated with the parameter $\lambda_{i}, i=1,2$, and assume that $\lambda_{1}>\lambda_{2}$. It is now clear from the proof of Theorem 4.2 that the value $V_{c, \lambda_{1}}(x)$ satisfies the variational inequality $\left(\mathcal{A} V_{c, \lambda_{1}}\right)(x)-r V_{c, \lambda_{1}}(x)+\pi(x) \leq 0$ for all $x \in \mathbb{R}_{+} \backslash\left\{y_{c, \lambda_{1}}^{*}\right\}$, where $y_{c, \lambda_{1}}^{*}$ denotes the optimal exercise threshold associated with the parameter $\lambda_{1}$. Moreover, since $V_{c, \lambda_{1}}(x)$ satisfies also the sufficient quasi-variational inequality

$$
\begin{aligned}
V_{c, \lambda_{1}}(x) & \geq \sup _{y \in[0, x]}\left[V_{c, \lambda_{1}}(y)+\frac{\lambda_{1}}{\beta}(x-y)\right]-c \\
& \geq \sup _{y \in[0, x]}\left[V_{c, \lambda_{1}}(y)+\frac{\lambda_{2}}{\beta}(x-y)\right]-c
\end{aligned}
$$

we find that $V_{c, \lambda_{1}}(x) \geq V_{c, \lambda_{2}}(x)$. Proving that $V_{c}(x)$ is a decreasing function of both the parameter $\beta$ and the sunk cost $c$ is entirely analogous.

Corollary 4.4 summarizes the impact of parametric changes on the value of the optimal policy. Unfortunately, it is difficult to characterize explicitly the impact of parametric changes in either $\lambda$ or $\beta$ on the optimal exercise boundary $y_{c}^{*}$ and the optimal generic initial state $y_{c}^{*}-\beta \zeta_{c}^{*}$. Fortunately, the impact of changes in the sunk cost $c$ can be explicitly characterized by studying the behavior of the implicit curves $I\left(y_{c}^{*}\right)-I\left(y_{c}^{*}-\beta \zeta_{c}^{*}\right)=0$ and 
$J\left(y_{c}^{*}\right)-J\left(y_{c}^{*}-\beta \zeta_{c}^{*}\right)=-\beta c$. Implicit differentiation of these curves with respect to $c$ together with the the relation $J^{\prime}(x)=-\psi(x) I^{\prime}(x)$ yield the conditions

$$
\frac{d\left(y_{c}^{*}-\beta \zeta_{c}^{*}\right)}{d c}=-\frac{\beta}{I^{\prime}\left(y_{c}^{*}-\beta \zeta_{c}^{*}\right)\left[\psi\left(y_{c}^{*}-\beta \zeta_{c}^{*}\right)-\psi\left(y_{c}^{*}\right)\right]}<0
$$

and

$$
\frac{d y_{c}^{*}}{d c}=-\frac{\beta}{I^{\prime}\left(y_{c}^{*}\right)\left[\psi\left(y_{c}^{*}-\beta \zeta_{c}^{*}\right)-\psi\left(y_{c}^{*}\right)\right]}>0 .
$$

In other words, the optimal threshold $y_{c}^{*}$ decreases, the regeneration state $y_{c}^{*}-$ $\beta \zeta_{c}^{*}$ increases and, therefore, the optimal impulse $\zeta_{c}^{*}$ decreases as the fixed intervention cost $c$ decreases. This observation is intuitively clear, since the proof of Lemma 4.1 implies that $\lim _{c \downarrow 0} y_{c}^{*}=\hat{x}$ and $\lim _{c \downarrow 0} \zeta_{c}^{*}=0$. Moreover, by continuity of the increasing fundamental solution $\psi(x)$, we discover that $\lim _{c \downarrow 0} \frac{d y_{c}^{*}}{d c}=\infty$ and $\lim _{c \downarrow 0} \frac{d \zeta_{c}^{*}}{d c}=-\infty$. Finally, by ordinary differentiation we find that

$$
\frac{d V_{c}}{d c}(x)=\left\{\begin{array}{ll}
\beta^{-1} J^{\prime}\left(y_{c}^{*}\right) \frac{d y_{c}^{*}}{d c} & x \geq y_{c}^{*} \\
-\beta^{-1} \psi(x) I^{\prime}\left(y_{c}^{*}\right) \frac{d y_{c}^{*}}{d c} & x<y_{c}^{*}
\end{array}<0 .\right.
$$

Summarizing, we formulate the following lemma characterizing the impact of the transaction $\operatorname{cost} c$ on the value of the optimal policy (see, for example, 22 for a similar observation).

Lemma 4.5. Assume, that the conditions of Lemma 4.1 are met. Then, $d\left(y_{c}^{*}-\right.$ $\left.\beta \zeta_{c}^{*}\right) / d c<0, d y_{c}^{*} / d c>0$, and $d \zeta_{c}^{*} / d c>0$. Moreover, $\lim _{c \downarrow 0} y_{c}^{*}=\hat{x}$, $\lim _{c \downarrow 0} \zeta_{c}^{*}=0, \lim _{c \downarrow 0} \frac{d y_{c}^{*}}{d c}=\infty, \lim _{c \downarrow 0} \frac{d \zeta_{c}^{*}}{d c}=-\infty$ and

$$
\lim _{c \downarrow 0} \frac{d V_{c}}{d c}(x)=-\infty
$$

for all $x \in \mathbb{R}_{+}$. 



\section{ILLUSTRATION: CONTROLLED GEOMETRIC BROWNIAN MOTION}

In order to illustrate our results explicitly, we now assume that the underlying controlled geometric Brownian motion evolves according to the dynamics characterized by the stochastic differential equation

$$
X_{t}^{\nu}=x+\int_{0}^{t} \mu X_{s}^{\nu} d s+\int_{0}^{t} \sigma X_{s}^{\nu} d W_{s}-\sum_{\tau_{k} \leq t} \beta \zeta_{k}, 0 \leq t \leq \tau_{0}^{\nu},
$$

where $\mu>0$ and $\sigma>0$ are exogenously determined known parameters. For the sake of the finiteness of the value of the considered stochastic control problems, we assume that $r>\mu$, that is, that the discount rate dominates the expected per capita growth rate of the controlled GBM. It is well-known that in this case the fundamental solutions read as $\psi(x)=x^{\kappa}$ and $\varphi(x)=x^{\phi}$, where

$$
\kappa=\frac{1}{2}-\frac{\mu}{\sigma^{2}}+\sqrt{\left(\frac{1}{2}-\frac{\mu}{\sigma^{2}}\right)^{2}+\frac{2 r}{\sigma^{2}}}>1
$$

and

$$
\phi=\frac{1}{2}-\frac{\mu}{\sigma^{2}}-\sqrt{\left(\frac{1}{2}-\frac{\mu}{\sigma^{2}}\right)^{2}+\frac{2 r}{\sigma^{2}}}<0 .
$$

Given the considered controlled process, we now assume that the revenue flow accrued from continuing operation reads as $\pi(x)=x^{\alpha}$, where $\alpha \in(0,1)$. Hence, we observe that $\theta(x)=\beta x^{\alpha}-(r-\mu) \lambda x$ implying that the conditions of Lemma 3.1 are satisfied and

$$
x^{*}=\operatorname{argmax}\{\theta(x)\}=\left(\frac{\alpha \beta}{(r-\mu) \lambda}\right)^{1 /(1-\alpha)} .
$$

Moreover, standard integration implies that $\left(R_{r} \pi\right)(x)=x^{\alpha} /(r-\delta(\alpha))$, where $\delta(\alpha)=\alpha \mu+\sigma^{2} \alpha(\alpha-1) / 2$.

The value of the optimal singular stochastic control policy reads as

$$
K(x)= \begin{cases}\frac{\lambda}{\beta}(x-\hat{x})+\frac{1}{r}\left(\hat{x}^{\alpha}+\frac{\lambda \mu}{\beta} \hat{x}\right) & x \geq \hat{x} \\ \frac{x^{\alpha}}{(r-\delta(\alpha))}+\frac{1}{\kappa}\left(\frac{\lambda}{\beta} \hat{x}-\frac{\alpha \hat{x}^{\alpha}}{(r-\delta(\alpha))}\right)\left(\frac{x}{\hat{x}}\right)^{\kappa} & x<\hat{x}\end{cases}
$$

where the optimal threshold $\hat{x}$ reads as

$$
\hat{x}=\left(\frac{\alpha \beta(\kappa-\alpha)}{(r-\delta(\alpha))(\kappa-1) \lambda}\right)^{1 /(1-\alpha)}=\left(1+\frac{1-\alpha}{\alpha-\phi}\right)^{1 /(1-\alpha)} x^{*}>x^{*} .
$$


Since

$$
\frac{\partial \phi}{\partial \sigma}=\frac{2 \phi(\phi-1)}{\sigma(\kappa-\phi)}>0
$$

we immediately find that

$$
\frac{\partial \hat{x}}{\partial \sigma}=\left(\frac{1-\phi}{\alpha-\phi}\right)^{\alpha /(1-\alpha)} \frac{x^{*}}{(\alpha-\phi)^{2}} \frac{\partial \phi}{\partial \sigma}>0 .
$$

Hence, we find that increased volatility increases the optimal threshold at which the irreversible policy should be exercised. Moreover, standard differentiation also yields that

$$
\frac{\partial \hat{x}}{\partial \beta}=\frac{\hat{x}}{(1-\alpha) \beta}>0 \quad \text { and } \quad \frac{\partial \hat{x}}{\partial \lambda}=-\frac{\hat{x}}{(1-\alpha) \lambda}<0
$$

demonstrating along the lines of our Corollary 3.4 that the optimal exercise threshold is an increasing function of the parameter $\beta$ and a decreasing function of the parameter $\lambda$. The optimal exercise boundary is illustrated as a function of the underlying volatility in Figure 1 for $\beta=0.9,1,1.1$ under the assumption that $r=0.045, \mu=0.025, \alpha=0.5$, and $\lambda=10$.

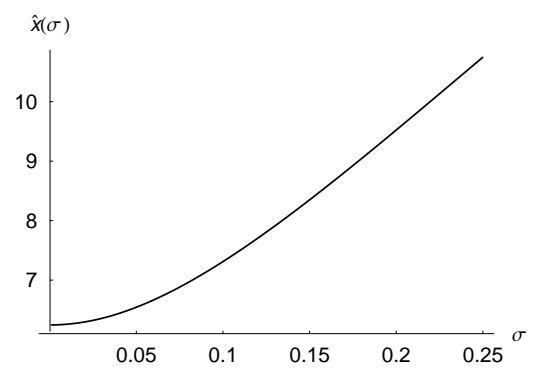

Figure 1: The optimal exercise boundary $\hat{x}(\sigma)$

The value of the associated optimal stopping problem reads as

$$
G_{c}(x)= \begin{cases}\frac{\lambda}{\beta} x-c & x \geq \bar{x}_{c} \\ \frac{x^{\alpha}}{(r-\delta(\alpha))}+\left(\frac{\lambda}{\beta} \bar{x}_{c}-\frac{\bar{x}_{c}^{\alpha}}{(r-\delta(\alpha))}-c\right)\left(\frac{x}{\bar{x}_{c}}\right)^{\kappa} & x<\bar{x}_{c}\end{cases}
$$

where the optimal stopping boundary $\bar{x}_{c}>\hat{x}$ is the unique root of the equation

$$
x^{\alpha}-\frac{(\kappa-1)(r-\delta(\alpha)) \lambda}{\beta(\kappa-\alpha)} x+\frac{\kappa c(r-\delta(\alpha))}{\kappa-\alpha}=0 .
$$


The optimal exercise boundary $\bar{x}_{c}$ is illustrated as a function of the underlying volatility in Figure 2 for $\beta=0.9,1,1.1$ under the assumption that $r=$ $0.045, \mu=0.025, \alpha=0.5, c=1$, and $\lambda=10$.

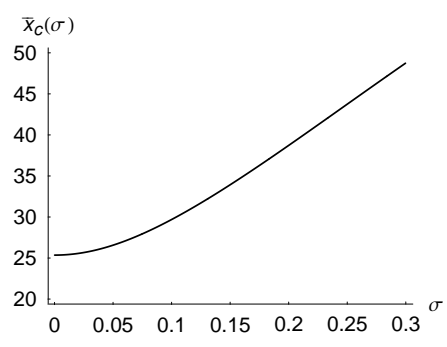

Figure 2: The optimal exercise boundary $\bar{x}_{c}(\sigma)$

The value of the considered stochastic impulse control problem reads as

$$
V_{c}(x)= \begin{cases}\frac{\lambda}{\beta}\left(x-y_{c}^{*}\right)+\frac{1}{\kappa}\left(\frac{(\kappa-\alpha) y_{c}^{* \alpha}}{(r-\delta(\alpha))}+\frac{\lambda}{\beta} y_{c}^{*}\right) & x \geq y_{c}^{*} \\ \frac{x^{\alpha}}{(r-\delta(\alpha))}-\frac{1}{\kappa}\left(\frac{\alpha y_{c}^{* \alpha}}{r-\delta(\alpha)}-\frac{\lambda}{\beta} y_{c}^{*}\right)\left(\frac{x}{y_{c}^{*}}\right)^{\kappa} & x<y_{c}^{*},\end{cases}
$$

where the optimal impulse threshold $y_{c}^{*}$ and generic initial state $z_{c}^{*}=y_{c}^{*}-\beta \zeta_{c}^{*}$ are the unique roots of the optimality conditions

$$
\alpha \beta\left(y_{c}^{* \alpha-\kappa}-z_{c}^{* \alpha-\kappa}\right)=(r-\delta(\alpha)) \lambda\left(y_{c}^{* 1-\kappa}-z_{c}^{* 1-\kappa}\right)
$$

and

$$
\beta(\kappa-\alpha)\left(y_{c}^{* \alpha}-z_{c}^{* \alpha}\right)-\lambda(r-\delta(\alpha))(\kappa-1)\left(y_{c}^{*}-z_{c}^{*}\right)=-\kappa \beta(r-\delta(\alpha)) c .
$$

Unfortunately, solving these non-linear equations explicitly is difficult (if possible at all). Hence, we illustrate numerically the optimal exercise threshold $y_{c}^{*}$, the optimal impulse $\zeta_{c}^{*}$, the ratio $\zeta_{c}^{*} / y_{c}^{*}$, and the optimal generic initial state $y_{c}^{*}-\zeta_{c}^{*}$ in Table 1 under the assumption that $r=0.045, \mu=0.025, \alpha=$ $0.5, c=1, \beta=1$, and $\lambda=10$.

Table 1: The optimal impulse control policy under the assumption that $r=0.045, \mu=0.025, \alpha=0.5, c=1, \beta=1$, and $\lambda=10$.

\begin{tabular}{|c|cccccc|}
\hline$\sigma$ & 0.01 & 0.05 & 0.1 & 0.15 & 0.2 & 0.25 \\
\hline$y_{c}^{*}$ & 9.697 & 10.168 & 11.443 & 13.196 & 15.222 & 17.399 \\
$\zeta_{c}^{*}$ & 5.215 & 5.511 & 6.319 & 7.447 & 8.780 & 10.252 \\
$\zeta_{c}^{*} / y_{c}^{*}$ & 0.5377 & 0.5420 & 0.5522 & 0.5643 & 0.5768 & 0.5892 \\
$y_{c}^{*}-\zeta_{c}^{*}$ & 4.482 & 4.656 & 5.124 & 5.749 & 6.443 & 7.147 \\
\hline
\end{tabular}


Table 1 clearly indicates that increased volatility does not only increase the optimal threshold at which the impulse policy is irreversibly exercised. It simultaneously increases both the size of the optimal policy and the optimal generic initial state. This result is of interest from the point of view of risk management since it clearly demonstrates that increased volatility will result both into a larger but less frequent dividend strategy and a larger generic initial capital protecting the rationally managed corporation from future unfavorable yet uncertain events (i.e. a larger capital buffer). It is also worth emphasizing that our results indicate that the dividend-capital-ratio $\zeta_{c}^{*} / y_{c}^{*}$ is also an increasing function of volatility. Consequently, even though the capital buffers $y_{c}^{*}-\zeta_{c}^{*}$ increase as volatility increases, the ratio between the buffers and the optimal capital decreases as volatility increases.

In order to analyze numerically the impact of a change in the parameter $\beta$ on the risk sensitivity of the optimal impulse policy numerically illustrated in Table 2 under the assumption that $r=0.045, \mu=0.025, \alpha=$ $0.5, c=1, \beta=1.1$, and $\lambda=10$ and in Table 3 under the assumption that $r=0.045, \mu=0.025, \alpha=0.5, c=1, \beta=0.9$, and $\lambda=10$. Along the lines of our previous findings on both the associated optimal stopping problem and the associated singular stochastic control problem, our numerical illustrations seem to indicate that the optimal variables are increasing as functions of the parameter $\beta$ and decreasing as functions of the parameter $\lambda$.

Table 2: The optimal impulse control policy under the assumption that $r=0.045, \mu=0.025, \alpha=0.5, c=1, \beta=1.1$, and $\lambda=10$.

\begin{tabular}{|c|cccccc|}
\hline$\sigma$ & 0.01 & 0.05 & 0.1 & 0.15 & 0.2 & 0.25 \\
\hline$y_{c}^{*}$ & 11.562 & 12.122 & 13.641 & 15.727 & 18.138 & 20.725 \\
$\zeta_{c}^{*}$ & 6.091 & 6.437 & 7.382 & 8.699 & 10.257 & 11.977 \\
$\zeta_{c}^{*} / y_{c}^{*}$ & 0.5268 & 0.5310 & 0.5412 & 0.5532 & 0.5655 & 0.5779 \\
$y_{c}^{*}-\zeta_{c}^{*}$ & 5.471 & 5.685 & 6.259 & 7.027 & 7.881 & 8.747 \\
\hline
\end{tabular}


Table 3: The optimal impulse control policy under the assumption that $r=0.045, \mu=0.025, \alpha=0.5, c=1, \beta=0.9$, and $\lambda=10$.

\begin{tabular}{|c|cccccc|}
\hline$\sigma$ & 0.01 & 0.05 & 0.1 & 0.15 & 0.2 & 0.25 \\
\hline$y_{c}^{*}$ & 7.989 & 8.377 & 9.429 & 10.876 & 12.55 & 14.35 \\
$\zeta_{c}^{*}$ & 4.394 & 4.643 & 5.323 & 6.272 & 7.394 & 8.635 \\
$\zeta_{c}^{*} / y_{c}^{*}$ & 0.55 & 0.5542 & 0.5646 & 0.5767 & 0.5892 & 0.6017 \\
$y_{c}^{*}-\zeta_{c}^{*}$ & 3.595 & 3.734 & 4.106 & 4.604 & 5.155 & 5.715 \\
\hline
\end{tabular}





\section{REFERENCES}

Alvarez, L. H. R. (1999) A Class of Solvable Singular Stochastic Control Problems, Stochastics \& Stochastics Reports, 67, 83 - 122.

Alvarez, L. H. R. (2001) Singular stochastic control, linear diffusions, and optimal stopping: A class of solvable problems, SIAM Journal on Control and Optimization, 39, 1697 - 1710.

Alvarez, L. H. R. (2004) Stochastic Forest Stand Value and Optimal Timber Harvesting, SIAM Journal on Control and Optimization, 42, 1972-1993.

Alvarez, L. H. R. (2004) A Class of solvable impulse control problems, Applied Mathematics \& Optimization, 49, 265-295.

Alvarez, L. H. R. and Koskela, E. (2003) A general approach to the stochastic rotation problem with amenity valuation, Department of Economics, University of Helsinki, Discussion papers, No 548.

Alvarez, L. H. R. and Koskela, E. (2004) Taxation and Rotation Age under Stochastic Forest Stand Value, Department of Economics, University of Helsinki, Discussion papers, No 604.

Alvarez, L. H. R. and Virtanen, J. (2004) A Class of Solvable Stochastic Dividend Optimization Problems: On the General Impact of Flexibility on Valuation, Turku School of Economics and Business Administration, Discussion Paper Series, nr 1/2004.

Bar-Ilan, A., Perry, D., and Stadje, W. (2004) A generalized impulse control model of cash management, Journal of Economic Dynamics \& Control, 28, 1013-1033.

Bensoussan, A. and Lions, J. L. (1984) Impulse Control and Quasivariational Inequalities, Gauthier- Villars, Paris.

Borodin, A. and Salminen, P. (2002) Handbook on Brownian motion - Facts and formulae, 2nd edition, Birkhauser, Basel.

Brekke, K. A. and Øksendal, B. (1996) A verification theorem for combined stochastic control and impulse control, Stochastic Analysis and Related Topics, VI, 211-220, Birkhäuser, Boston. 
Brekke, K. A. and Øksendal, B. (1994) Optimal switching in an economic activity under uncertainty, SIAM Journal on Control and Optimization, 32, $1021-1036$.

Cadenillas, A., Sarkar, S., and Zapatero, F. (2003) Optimal dividend policy with mean reverting cash reservoir, Working Paper.

Freidlin, M. (1985) Functional integration and partial differential equations, Princeton UP, Princeton.

Harrison, J. M., Sellke, T. M., and Taylor, A. J. (1983) Impulse control of Brownian motion, Mathematics of Operations Research, 8, 454-466.

Kobila, Т. Ø. (1993) A class of solvable stochastic investment problems involving singular controls, Stochastics and Stochastics Reports, 43, 29-63.

Korn, R. (1999) Some applications of impulse control in mathematical finance, Mathematical Methods of Operations Research, 50, 493 - 518.

Menaldi, J. L. and Rofman, E. (1983) On stochastic control problems with impulse cost vanishing, Lecture notes in economics and mathematical systems, 215, Fiacco, A. V. and Kortanek, K. O. (eds.), Springer-Verlag, Berlin, 771-802.

Mundaca, G. and Øksendal, B. (1998) Optimal stochastic intervention control with application to the exchange rate, Journal of Mathematical Economics, 29, 225-243.

Øksendal, A. (2000) A semi-group approach to impulse control problems, University of Oslo, Department of Mathematics, Preprint series \#14.

Øksendal, A. (2000) Irreversible Investment Problems, Finance \& Stochastics, 4, 223-250.

Øksendal, B. (1999) Stochastic control problems where small intervention costs have big effects, Applied mathematics \& Optimization, 40, 355-375.

Øksendal, B. (2003) Stochastic differential equations: An introduction with applications, Sixth Edition, Springer, Berlin.

Peura, S. and Keppo, J. S. (2003) Optimal Bank Capital with Costly Recapitalization, EFMA 2003 Helsinki Meetings. 
Protter, P. (1990) Stochastic integration and differential equations, Springer, New York.

Sødal, S. (2002) The stochastic rotation problem: A comment, Journal of Economic Dynamics and Control, 26, 509-515.

Willassen, Y. (1998) The stochastic rotation problem: A generalization of Faustmann's formula to stochastic forest growth, Journal of Economic Dynamics and Control, 22, 573-596. 